\title{
FRUSTRATION IN FERROMAGNETIC MATERIALS
}

\author{
By \\ R.D. James \\ and \\ D. Kinderlehrer
}

IMA Preprint Series \# 643

May 1990 


\title{
Frustration in Ferromagnetic Materials
}

\author{
R. D. James ${ }^{\dagger}$ and D. Kinderlehrer ${ }^{\ddagger}$
}

Dedicated to the memory of Stella Dafermos

\section{Table of Contents}

1. Introduction ............................................................... 1

2. Energy of Ferromagnetic Materials................................................ 4

3. The Minimum of the Functional ............................................. 9

4. Attainment of the Minimum .................................................... 14

(a) Uniaxial case .......................................................... 15

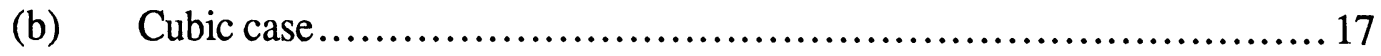

5. Further Analysis of Domain Structures...................................... 21

6. Bound on the Minimum Energy via a Lagrangian Formulation......................2 27

7. Effect of a Constant Applied Field ...................................... 31

8. Remarks on the Calculations of Brown and Lifshitz......................... 34

\section{Introduction}

Predictions of the domain structure of real ferromagnetic materials are usually derived from either domain theory or micromagnetics. Domain theory, often favored by experimentalists, has its origins in the famous 1935 paper of Landau and Lifshitz. Landau and Lifshitz [1935] calculated the energy of a domain wall dividing an infinite cylinder with magnetization vector m depending smoothly on only the axial variable. They accounted for exchange energy, the energy that arises

\footnotetext{
$\dagger$ Department of Aerospace Engineering and Mechanics and

\$ School of Mathematics, University of Minnesota, Minneapolis, MN 55455, members of the research group on Transitions and Defects in Ordered Materials, to appear in Continuum Mechanics and Thermodynamics
} 
from gradients of the magnetization, and for anisotropy energy, the energy that favors axial magnetization, but not for magnetostatic energy. The main result of their calculation is an expression for the energy stored in the region of rapid variation of $\mathrm{m}$, i.e., the interfacial energy of the domain wall.

Domain theory takes the expression of the interfacial energy from the calculation of Landau and Lifshitz, or one of the improvements which accounts better for the wall structure*, and assigns this energy to sharp discontinuities of magnetization. In practice, domain theorists begin with a divergence-free field of magnetization having a certain specific arrangement of interfaces specified by several parameters, and then adjust the values of these parameters so as to minimize energy.

W.F. Brown [1962] criticized domain theory on the grounds that it contains too many geometric restrictions. He remarks that "The mere existence of a lower energy configuration does not guarantee that that configuration will be attained; if it did, there would be no such phenomenon as hysteresis. Second, the particular configuration devised is dependent on the ingenuity of the theorist who devised it; conceivably a more ingenious theorist could devise one with even lower free energy." Brown introduced an alternative approach termed micromagnetics that avoids the geometric restrictions. The theory of micromagnetics develops an expression for the free energy of a general magnetization field and then seeks to determine that field so as to minimize energy in an appropriate space.

Despite the general attractiveness of Brown's philosophy, micromagnetics has not gained general acceptance. This is apparently due to two features of micromagnetics. First, in the case often considered (e.g., Brown [1962]) with exchange energy omitted, the minimum of the free energy is not generally attained. Minimizing sequences for the energy exhibit finer and finer structure. Nonattainment of the minimum does not occur for all crystal symmetries, and we

\footnotetext{
${ }^{*}$ See Kléman [1983] for a discussion of different wall models.
} 
suggest that this explains in some way the huge dichotomy of scales exhibited by ferromagnetic materials, whereby large cubic ferromagnets may exhibit a few huge domains while large uniaxial ferromagnets always exhibit relatively fine columnar or laminar domains. We show this in Sections 3 and 4. Second, we generalize in Section 8 a metastability calculation of Brown that also seems to be an origin of the distrust of micromagnetics. The calculation leads to the so-called "coercivity paradox." Our calculation sheds light on the coercivity paradox by showing precisely when the metastable state becomes unstable relative to finite disturbances.

Martensitic materials also exhibit extremely fine twinned microstructures often appearing as layers or layers within layers. In recent years a new theory of martensite has been developed which involves free energies that do not have attained minima. See, e.g., Ball and James [1987], Chipot and Kinderlehrer [1988], Fonseca [1988], James and Kinderlehrer [1990], Pedregal [1989] and Kohn [1989]. The theory of martensite that has emerged is in many ways analogous to micromagnetics without exchange energy (with one significant difference described below) and the analogy can be stretched to include the crystallographic theory of martensite, the analog of domain theory for the martensitic materials. As in the martensite, a study of the minimizing sequences (Sections 2 through 5 below) gives a rather complete picture of the macroscopic aspects of the domain structure, and is particularly useful for predicting where in the body fine structure will occur, in addition to the averaged properties of this fine structure. It is also anticipated that information on where fine structure must occur will be useful in setting up reliable micromagnetic computations with exchange energy included, such as those under development by Luskin.

A remarkable feature of ferromagnetic materials is that the single domain state is generally unstable. This contrasts with martensite, where the single variant configuration is stable for arbitrarily large samples. In other physical systems, such as the blue phase of cholesteric liquid crystals, the failure of stability of the uniform state relative to an array of defects is termed "frustration." Our calculations could be interpreted as one possible interpretation of this phenomenon at a macroscopic scale. The frustration in our system arises from the competition of 
an anisotropy energy which demands constant magnetization strength with an induced field energy which prefers to tend to zero. A consequence of this is to promote development of a fine scale structure which seeks to compromise the constraint of constant magnetization strength. A different mechanism is given by Sethna [1987] for the blue phase. He associates the term frustration with the failure of existence of a pointwise minimizer of the energy density, such as occurs in the problem

$$
\min \int_{\Omega}|\nabla \mathrm{u}-\mathrm{F}|^{2} \mathrm{dx}
$$

where $F(x)$ is a smooth vector valued function which is not a gradient. This agrees with our interpretation in the case of zero applied field and in some other special cases, but differs from our interpretation in that his energy functional does have an attained absolute minimum in an ordinary function space whereas ours generally does not.

\section{Energy of Ferromagnetic Materials}

The conventional theory of ferromagnetic materials is based on the classical assumption of Weiss, Landau and Lifshitz that the magnetization $\mathrm{m}$ varies with position $\mathrm{x} \in \Omega$ but has a fixed, temperature dependent magnitude:

$$
|\mathrm{m}(\mathrm{x})|=\mathrm{f}(\mathrm{T}), \quad \mathrm{x} \in \Omega
$$

with $f(T)=0$ for $T \geq T_{c}, T_{c}$ being the Curie point. In this paper we shall not vary the temperature so, without loss of generality, we shall consider vector fields

$$
\mathrm{m}: \Omega \rightarrow \mathrm{s}^{2}
$$


the unit sphere in $\mathbb{R}^{3}$, or, more generally, with $\Omega \subset \mathbb{R}^{\mathbf{n}}$ and

$\mathrm{m}: \Omega \rightarrow \mathrm{S}^{\mathrm{n}-1}$

The energy of a rigid ferromagnetic material is assumed to consist of the sum of three parts (cf. Brown [1963], Landau and Lifshitz [1984]). The exchange energy models the tendency of neighboring magnetic moments of atoms to align and has the form

$$
\int_{\Omega} \nabla \mathrm{m} \cdot \mathrm{A} \nabla \mathrm{mdx}
$$

where $\mathrm{A}$ is a linear transformation on constant $3 \times 3$ matrices. The anisotropy energy models the tendency of the magnetization to point in specific crystallographic directions and is given by an even function $\varphi(\mathrm{m})$ which exhibits crystallographic symmetry. We shall discuss two cases:

(i) Cubic case. There are orthonormal vectors $\left\{\mathrm{m}_{\mathrm{i}}\right\}$ such that*

$$
\begin{aligned}
0= & \varphi\left( \pm m_{1}\right)=\varphi\left( \pm m_{2}\right)=\varphi\left( \pm m_{3}\right)<\varphi(m) \text { for all } \\
& m \notin\left\{ \pm m_{1}, \pm m_{2}, \pm m_{3}\right\} .
\end{aligned}
$$

(ii) Uniaxial case

$$
0=\varphi\left( \pm \mathrm{m}_{1}\right)<\varphi(\mathrm{m}) \text { for all } \mathrm{m} \neq \pm \mathrm{m}_{1} \text {. }
$$

Without loss of generality we have made the minimum values of $\varphi$ equal to zero. Finally, the magnetostatic energy is the energy of the magnetostatic field set up by the magnetization $\mathrm{m}$. The

\footnotetext{
* This is the simplest assumption appropriate to cubic symmetry. It corresponds to having the easy axis along (100) directions such as in iron. Other cubic materials such as nickel have a greater number of minima. Because $\varphi$ exhibits crystallographic symmetry, it always has a set of minimizers of the form (orbit P)e where $\mathrm{P}$ is the point group of the material.
} 
form of this energy is calculated, for example, by identifying $m$ with the quantity $(i / c) d a$ where $i$ is the current in a plane filamentary circuit of vector-area da and then by regarding $\Omega$ as a continuum field of such circuits. The form of the magnetostatic energy is

$$
\int_{\mathbb{R}}|\nabla \mathrm{u}|^{2} \mathrm{dx}
$$

where

$$
\operatorname{div}\left[-\nabla \mathrm{u}+\mathrm{m} \chi_{\Omega}\right]=0 \text { on } \mathbb{R}^{3}
$$

Here, the presence of the term $m \chi_{\Omega}$ is a reminder that (2.7) is solved on all of $\mathbb{R}^{3}$ but with $\mathrm{m} \chi_{\Omega}$ $=0$ on $\mathbb{R}^{3}-\Omega$. Equation (2.7) arises from the two Maxwell's equations

$$
\begin{aligned}
& \operatorname{div} B=0 \\
& \operatorname{curl} H=0
\end{aligned}
$$

and the definition (omitting unessential constants),

$$
\mathrm{B}=\mathrm{H}+\mathrm{m} \text {. }
$$

Using (2.8), we have introduced the potential $u$ with $H=-\nabla u$. Thus, the total energy is formally

$$
E_{1}(m)=\int_{\Omega}[\nabla m \cdot A \nabla m+\varphi(m)] d x+\frac{1}{2} \int_{\mathbb{R}^{3}}|\nabla u|^{2} d x
$$

Here $\mathrm{u}$ is obtained from $\mathrm{m}$ by solving (2.7) subject to the appropriate conditions at $\infty$ (see Section 3). 
An odd feature of the constitutive part of this energy, namely, the first two terms of (2.9), is that it does not embody the most general frame-indifferent energy of the form $\varphi\left(\nabla \mathrm{m}, \mathrm{m}, \mathrm{e}_{\mathrm{i}}\right)$, which would seem to represent the minimal assumption. Here, for a rigid crystal, $\left\{\mathrm{e}_{1}, \mathrm{e}_{2}, \mathrm{e}_{3}\right\}$ denote lattice vectors of the crystal and for a rigid ferromagnet are restricted to lie in the domain $\mathrm{SO}(3) \hat{\mathrm{e}}_{\mathrm{i}}$ with $\hat{\mathrm{e}}_{\mathrm{i}}$ constant, linearly independent vectors. Frame indifference would require that

$$
\varphi\left(\mathrm{R} \nabla \mathrm{mR}^{\mathrm{T}}, \mathrm{Rm}, \mathrm{Re}_{\mathrm{i}}\right)=\varphi\left(\nabla \mathrm{m}, \mathrm{m}, \mathrm{e}_{\mathrm{i}}\right) \quad \text { for all } \mathrm{R} \in \mathrm{SO}(3)
$$

Hence, even frame-indifferent quadratic terms normally found in the energy for liquid crystals, for example (Frank [1958])

$$
\kappa_{2}(m \cdot \operatorname{curl} m+q)+\kappa_{3}|m \wedge \operatorname{curl} m|^{2},
$$

are missing from (2.9). It is possible that the molecular theories used to derive (2.9) contain hidden geometric assumptions which forbid certain interactions, such as was the case in the theory of liquid crystals before the appearance of Frank's paper referenced above. We shall not pursue this issue here.

The exchange energy can be thought of as giving rise to a surface energy on domain boundaries. The calculation which justifies this fact in an asymptotic sense is given in a recent paper of Anzellotti, Baldo and Visintin [1989]. Their calculation qualitatively is similar to the calculation of the asymptotic behavior of minima for a van der Wall's fluid with surface energy measured by the volume integral of $|\nabla v|^{2}$ where $\mathrm{v}$ is the specific volume, $c f$. Kohn and Sternberg [1989]. The scaling used in these papers $-\varepsilon$ in front of the exchange energy and $\varepsilon^{-1}$ in front of the anisotropy energy with $\varepsilon \rightarrow 0-$ might be inferred from the [1935] paper of Landau and Lifshitz in the magnetic case. 
Except in certain situations which we treat explicitly in Section $5 b$, crystals of $\mathrm{mm}$ or greater size exhibit fine domain structures, either fine bands in the material or coarse bands that show splitting into finer and finer domains at the surface of the crystal. In these cases the crystal exhibits a large surface area of domain walls, suggesting that the essential domain structure can be obtained by omitting the exchange energy. A similar point of view in theories for martensitic materials has been successful in predicting their twinned structures and macroscopic properties ( $c f$. Ball and James [1987, 1990], James and Kinderlehrer [1989]). The operating principle in those calculations has been that the surface energy only selects some (fine) scale while the minimization of bulk energy determines the possible microstructures on that scale. A major advantage of this approach is that detailed stable domain patterns in a great many cases can be calculated rigorously without resorting to approximate methods. This viewpoint has also been useful in setting up reliable computations of domain patterns in martensitic materials, such as those of Collins and Luskin [1989], which necessarily must cope with domain refinement. Analyses of the type presented here are particularly helpful for deciding where in the body one should expect to find fine domain structures.

To explore this idea further, we shall put $A=0$ in (2.9). Set

$$
E_{0}(m)=\int_{\Omega} \varphi(m) d x+\frac{1}{2} \int_{\mathbb{R}^{3}}|\nabla u|^{2} d x
$$

subject to

$$
\operatorname{div}\left(-\nabla \mathrm{u}+\mathrm{m} \chi_{\Omega}\right)=0 \text { in } \mathbb{R}^{3}
$$

and consider

$$
\inf _{|m|=1} E_{0}(m) \text {. }
$$


Observe that there is an alternative expression for (2.11). Since (2.12) means that

$$
\int_{\mathbb{R}}\left(-\nabla \mathrm{u}+\mathrm{m} \chi_{\Omega}\right) \cdot \nabla \zeta \mathrm{dx}=0
$$

whenever $\nabla \zeta \in \mathrm{L}^{2}\left(\mathbb{R}^{3}\right)$, then if we set $\zeta=\mathrm{u}$ we get

$$
\int_{\mathbb{R}^{3}}|\nabla \mathrm{u}|^{2} \mathrm{dx}=\int_{\mathbb{R}^{3}} \mathrm{~m} \chi_{\Omega} \cdot \nabla \mathrm{udx} .
$$

Hence

$$
\mathrm{E}_{\mathrm{o}}(\mathrm{m})=\int_{\Omega} \varphi(\mathrm{m}) \mathrm{dx}+\frac{1}{2} \int_{\Omega} \nabla \mathrm{u} \cdot \mathrm{m} \mathrm{dx}
$$

\section{The Minimum of the Functional}

From (2.11) it is clear that $E_{0} \geq 0$. In this section we show that

$$
\inf _{|\mathrm{m}|=1} \mathrm{E}_{\mathrm{o}}(\mathrm{m})=0
$$

provided $\varphi$ has minimizers of the form $\pm m_{1}$. Recall that inf $\varphi=0$. This covers both the uniaxial and cubic symmetry hypotheses, (2.4) and (2.5). First let us verify that $E_{0}(m)$ is well-defined. In particular, we check the sense in which we understand the equation (2.12). Throughout this paper we make the standing assumption that $\Omega$ is open and bounded and has a Lipschitz boundary.

Let $\mathrm{B} \subset \mathbb{R}^{\mathrm{n}}$ be a fixed ball with $\bar{\Omega} \subset \mathrm{B}$; suppose $\mathrm{n}>2$, and let

$$
\mathrm{V}=\left\{\mathrm{v} \in \mathrm{H}^{1}(\mathrm{~B}): \nabla \mathrm{v} \in \mathrm{L}^{2}\left(\mathbb{R}^{\mathrm{n}}\right) \text { and } \int_{\mathrm{B}} \mathrm{v} \mathrm{dx}=0\right\}
$$


$\mathrm{V}$ is a Hilbert space with inner product

$$
(\mathrm{u}, \mathrm{v})=\int_{\mathbb{R}^{\mathrm{n}}} \nabla \mathrm{u} \cdot \nabla \mathrm{vdx}+\int_{\mathrm{B}} \mathrm{uvdx}
$$

By Poincaré's inequality,

$$
\int_{B} u^{2} d x \leq c \int_{B}|\nabla u|^{2} d x \leq c \int_{\mathbb{R}^{n}}|\nabla u|^{2} d x, u \in V,
$$

from which it follows that a norm equivalent to $(v, v)^{1 / 2}$ on $\mathrm{V}$ is given by

$$
\left(\int_{\mathbb{R}^{\mathrm{n}}}|\nabla \mathrm{u}|^{2} \mathrm{dx}\right)^{1 / 2}
$$

We shall regard (3.4) as the norm on $\mathrm{V}$.

Lemma 3.1. Let $\mathrm{m} \in \mathrm{L}^{2}\left(\Omega ; \mathbb{R}^{\mathrm{n}}\right)$. The equation

$$
\mathrm{u} \in \mathrm{V}: \operatorname{div}\left(-\nabla \mathrm{u}+\mathrm{m} \chi_{\Omega}\right)=0
$$

admits a unique solution in V. The mapping

$$
\begin{aligned}
\mathrm{T}: \mathrm{L}^{2}\left(\Omega ; \mathbb{R}^{\mathrm{n}}\right) & \rightarrow \mathrm{V} \\
\mathrm{m} & \rightarrow \mathrm{u}
\end{aligned}
$$

is linear and continuous.

The equation (3.5) means that

$$
\int_{\mathbb{R}^{\mathrm{n}}}\left(-\nabla \mathrm{u}+\mathrm{m} \chi_{\Omega}\right) \cdot \nabla \zeta \mathrm{dx}=0 \quad \text { for } \zeta \in \mathrm{V} .
$$


Proof. The functional

$$
\mathrm{I}(\mathrm{v})=\frac{1}{2} \int_{\mathbb{R}^{\mathrm{n}}}|\nabla \mathrm{v}|^{2} \mathrm{dx}+\int_{\mathbb{R}^{\mathrm{n}}} \nabla \mathrm{v} \cdot \mathrm{m} \chi_{\Omega} \mathrm{dx}, \quad \mathrm{u} \in \mathrm{V},
$$

is convex and lower semicontinuous with respect to weak convergence in $\mathrm{V}$. Owing to the elementary estimate

$$
\mathrm{I}(\mathrm{v}) \geq\left(\frac{1}{2}-\varepsilon\right) \int_{\mathbb{R}^{\mathrm{n}}}|\nabla \mathrm{v}|^{2} \mathrm{dx}-\frac{\varepsilon}{2} \int_{\Omega}|\mathrm{m}|^{2} \mathrm{dx},
$$

it is bounded below. $\mathrm{T}$ admits a unique minimizer in $\mathrm{V}$ and this minimizer satisfies the Euler equations, (3.5). If $\mathrm{m}, \mathrm{m}^{\prime} \in \mathrm{L}^{2}(\Omega)$ have solutions $\mathrm{u}, \mathrm{u}^{\prime} \in \mathrm{V}$ respectively, setting $\zeta=\mathrm{u}-\mathrm{u}^{\prime}$ in (3.6), subtracting, and applying the Schwarz inequality gives that

$$
\left\|\mathrm{u}-\mathrm{u}^{\prime}\right\|_{\mathrm{V}} \leq \mathrm{c}\left\|\mathrm{m}-\mathrm{m}^{\prime}\right\|_{\mathrm{L}^{2}}
$$

It follows from general principles, and is easy to check, that $T$ is also continuous from $\mathrm{L}^{2}\left(\Omega ; \mathbb{R}^{\mathrm{n}}\right)$ in the weak topology to $\mathrm{V}$ in the weak topology. Hence if

$$
\begin{aligned}
& \mathrm{m}^{\mathrm{k}} \rightarrow \mathrm{m} \quad \text { in } \mathrm{L}^{2}\left(\Omega ; \mathbb{R}^{\mathrm{n}}\right) \text { weakly, then } \\
& \nabla \mathrm{u}^{\mathrm{k}} \rightarrow \nabla \mathrm{u} \text { in } \mathrm{L}^{2}\left(\mathbb{R}^{\mathrm{n}} ; \mathbb{R}^{\mathrm{n}}\right) \text { weakly }
\end{aligned}
$$

By the Rellich and trace theorems, it then follows that

$$
\mathrm{u}^{\mathrm{k}} \rightarrow \mathrm{u} \text { in } \mathrm{L}^{2}(\Omega) \text { and } \mathrm{L}^{2}(\partial \Omega)
$$

In fact, owing to the compact support of $\mathrm{m}$, (3.5) admits a solution in $\mathrm{H}^{1}\left(\mathbb{R}^{\mathrm{n}}\right)$. The proof of this, although not difficult, is not germane to our considerations here. We turn now to the 


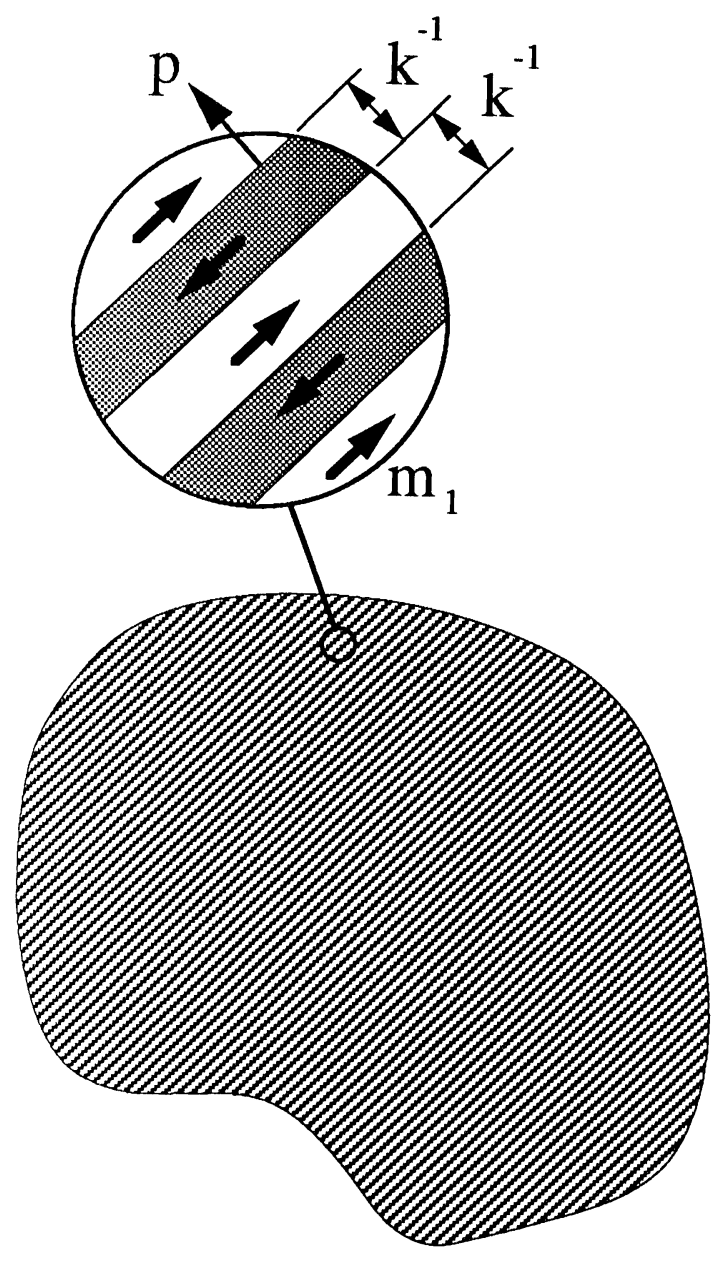

a.

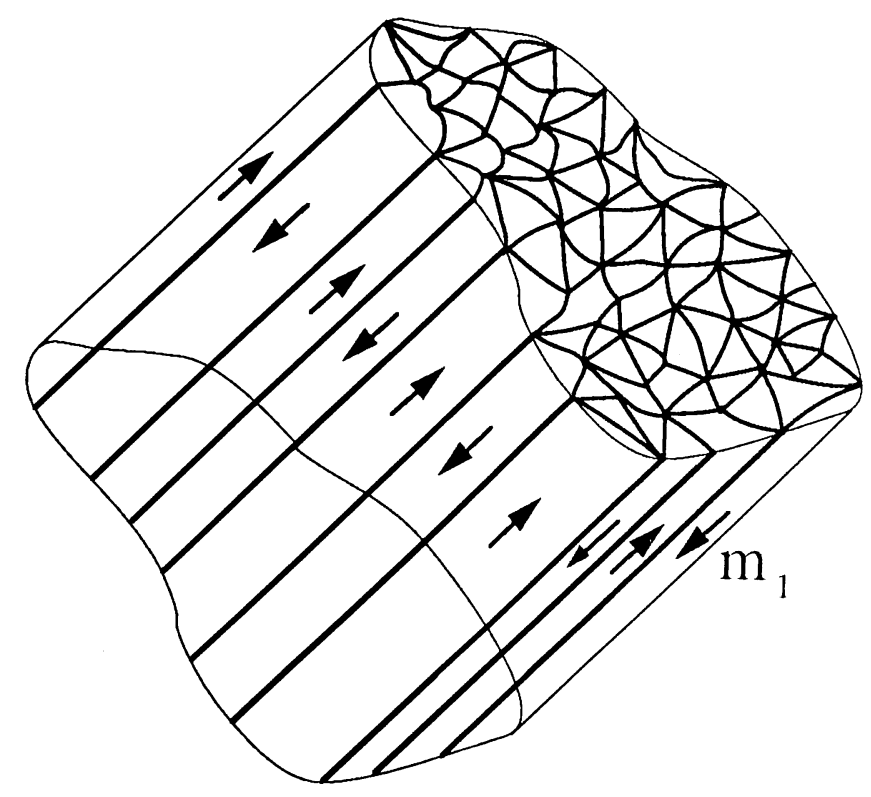

b.

Figure 1. Construction for the minimizing sequence. 
proof of (3.1). Assume that $\varphi\left(\mathrm{m}_{1}\right)=\varphi\left(-\mathrm{m}_{1}\right)=0$, and choose $\mathrm{p} \in \mathbb{R}^{\mathrm{n}}$ with $\mathrm{p} \cdot \mathrm{m}_{1}=0$. Let $\theta: \mathbb{R} \rightarrow \mathbb{R}$ be periodic of period 1 with

$$
\theta(t)=\left\{\begin{array}{cc}
1 & t \in[0,1 / 2) \\
-1 & t \in[1 / 2,1)
\end{array}\right. \text {. }
$$

Referring to Figure 1a, set

$$
\mathrm{m}^{\mathrm{k}}(\mathrm{x})= \begin{cases}\mathrm{m}_{1} \theta(\mathrm{kp} \cdot \mathrm{x}) & \mathrm{x} \in \Omega, \\ 0 & \mathrm{x} \in \mathbb{R}^{\mathrm{n}_{-} \Omega},\end{cases}
$$

and note that $\varphi\left(\mathrm{m}^{\mathrm{k}}\right)=0$.

We assert two properties of $\mathrm{m}^{\mathrm{k}}$ :

(a) $\quad \operatorname{div} \mathrm{m}^{\mathrm{k}}=0$ in $\Omega$

This is because $m_{1} \cdot p=0$.

(b) $\quad \mathrm{m}^{\mathrm{k}} \rightarrow 0$ in $\mathrm{L}^{2}\left(\mathbb{R}^{\mathrm{n}}\right)$ weakly.

This is standard. It suffices to show that for any cube D,

$$
\lim _{k \rightarrow \infty} \int_{D} m^{k} d x=0
$$

Now let $\mathrm{u}^{\mathrm{k}}$ be the solution of (3.5) corresponding to $\mathrm{m}^{\mathrm{k}}$. Then by (3.8)

$$
\mathrm{u}^{\mathrm{k}} \rightarrow 0 \text { in } \mathrm{V} \text { weakly, }
$$




$$
\mathrm{u}^{\mathrm{k}} \rightarrow 0 \text { in } \mathrm{L}^{2}(\Omega) \text { and in } \mathrm{L}^{2}(\partial \Omega)
$$

Now we may calculate

$$
\begin{aligned}
\mathrm{E}_{0}\left(\mathrm{~m}^{\mathrm{k}}\right) & =\int_{\Omega} \varphi\left(\mathrm{m}^{\mathrm{k}}\right) \mathrm{dx}+\frac{1}{2} \int_{\Omega} \mathrm{m}^{\mathrm{k} \cdot \nabla \mathrm{u}^{\mathrm{k}} \mathrm{dx}} \\
& =-\frac{1}{2} \int_{\Omega} \operatorname{div} \mathrm{m}^{\mathrm{k}} \mathrm{u}^{\mathrm{k}} \mathrm{dx}+\frac{1}{2} \int_{\Omega} \mathrm{m}^{\mathrm{k} \cdot v} \mathrm{u}^{\mathrm{k}} \mathrm{d} S \\
& =+\frac{1}{2} \int_{\partial} \mathrm{m}^{\mathrm{k} \cdot v \mathrm{u}^{\mathrm{k}} \mathrm{d} S} \\
& \rightarrow 0 \text { as } \mathrm{k} \rightarrow \infty .
\end{aligned}
$$

Obviously there are many other choices of minimizing sequences for $E_{0}$. For example, fine columnar domains as pictured in Figure $1 \mathrm{~b}$, as long as they have the property that they have equal volumes on average, generate a minimizing sequence. Uniaxial materials often have the general kind of domain structure pictured in Figure 1b. A great variety of cross-sectional shapes are observed as shown, for example, by Carey and Isaac [1966] or Chikazumi [1964]. On the other hand, there are restrictions on the minimizing sequences. We explore these in Section 5.

\section{Attainment of the Minimum}

We turn now to the question of whether of not the infimum

$$
\mathrm{E}_{\mathrm{o}}=0
$$

is attained by an $\mathrm{m} \in \mathrm{L}^{2}\left(\Omega ; \mathrm{S}^{\mathrm{n}-1}\right)$. The answer is different in the uniaxial and cubic cases. The former will exhibit the frustration described in the introduction while the latter admits a solution.

If $m \in L^{2}\left(\Omega ; S^{n-1}\right)$ with 


$$
\mathrm{E}_{0}(\mathrm{~m})=0
$$

then

$$
\mathrm{m}(\mathrm{x}) \in \mathrm{K}:=\{\mathrm{m}: \varphi(\mathrm{m})=0,|\mathrm{~m}|=1\} \text { in } \Omega \text { a.e. }
$$

and the corresponding magnetostatic potential u vanishes identically. By (3.5)

$$
\operatorname{div} m \chi_{\Omega}=0 \text { in } \mathbb{R}^{n},
$$

that is,

$$
\int_{\mathbb{R}^{\mathrm{n}}} \mathrm{m} \chi_{\Omega} \cdot \nabla \zeta \mathrm{dx}=0 \quad \text { for all } \quad \zeta \in \mathrm{V}
$$

(a) Uniaxial case

We show here that $E_{0}$ does not attain its minimum.

Lemma 4.1. Suppose that $\mathrm{f} \in \mathrm{L}^{2}\left(\mathbb{R}^{\mathrm{n}}\right)$ with supp $\mathrm{f} \subset \mathbb{R}^{\mathrm{n}}$ compact. Then the mapping from $\mathrm{C}^{\infty}\left(\mathbb{R}^{\mathrm{n}}\right)$ to $\mathbb{R}^{\mathrm{n}}$ given by

$$
\zeta \rightarrow \int_{\mathbb{R}^{\mathrm{n}}} \mathrm{f} \nabla \zeta \mathrm{dx}
$$

either has rank $\mathrm{n}$ or $\mathrm{f}$ vanishes identically.

Proof. Suppose the rank of the mapping in (4.4) is less than $\mathrm{n}$. Then there is a (unit) vector $\xi \in$ $\mathbb{R}^{\mathrm{n}}$ such that

$$
\int_{\mathbb{R}^{n}} \mathrm{f} \nabla \zeta \cdot \xi \mathrm{dx}=0 \quad \text { for } \zeta \in \mathrm{C}^{\infty}\left(\mathbb{R}^{\mathrm{n}}\right)
$$


or

$$
\int_{\mathbb{R}^{n}} \mathrm{f} \frac{\partial \zeta}{\partial \xi} \mathrm{dx}=0 \quad \text { for } \zeta \in \mathrm{C}^{\infty}\left(\mathbb{R}^{\mathrm{n}}\right)
$$

Without loss of generality, assume that $\xi=\mathrm{e}_{\mathrm{n}}$. Then by Fubini's Theorem and the Lemma of duBois Reymond it follows that $\mathrm{f}=\mathrm{f}\left(\mathrm{x}_{1}, \ldots, \mathrm{x}_{\mathrm{n}-1}\right)$, a function of $\mathrm{n}-1$ variables. Hence if supp $\mathrm{f}$ is compact, f vanishes identically .

Assume as above that $\{\mathrm{m}: \varphi(\mathrm{m})=0,|\mathrm{~m}|=1\}=\left\{ \pm \mathrm{m}_{1}\right\}$, has two points and that (4.1) holds. Then there is

$$
\begin{aligned}
\mathrm{m} & =\chi_{\mathrm{A} \mathrm{m}_{1}}-\chi_{\Omega-\mathrm{Am}_{1}} \\
& =\left[\chi_{\mathrm{A}}-\left(\chi_{\Omega}-\chi_{\mathrm{A}}\right)\right] \mathrm{m}_{1} \\
& =\left(2 \chi_{\mathrm{A}}-\chi_{\Omega}\right) \mathrm{m}_{1}
\end{aligned}
$$

and

$$
\operatorname{div} m=0 .
$$

Hence

$$
0=\int_{\mathbb{R}^{\mathrm{n}}} \mathrm{m} \cdot \nabla \zeta \mathrm{dx}=\int_{\mathbb{R}^{\mathrm{n}}}\left(2 \chi_{\mathrm{A}}-\chi_{\Omega}\right) \mathrm{m}_{1} \cdot \nabla \zeta \mathrm{dx}
$$

Thus, with $\mathrm{f}=2 \chi_{\mathrm{A}}-\chi_{\Omega}$, the mapping

$$
\zeta \rightarrow \int_{\mathbb{R}^{\mathrm{n}}} \mathrm{f} \nabla \zeta \mathrm{dx}
$$


does not have full rank, so $f=0$, or $|\Omega|=0$, which violates our hypothesis about $\Omega$. Thus, in the uniaxial case the minimum is not attained in $\mathrm{V}$.

This argument is sensitive to the form of $\varphi$. For example, if we consider a function $\varphi(m, x)$ with explicit dependence on $x$ appropriate to a "locally uniaxial" crystal obtained by bending a uniaxial crystal into the shape of a ring, e.g.

$$
\varphi(\mathrm{m}(\mathrm{x}), \mathrm{x}) \leq \varphi(\mathrm{m}, \mathrm{x}) \quad \mathrm{x} \in \mathrm{S}^{1} \times \mathrm{S}^{1}, \text { for all }|\mathrm{m}|=1
$$

where

$$
\mathrm{m}(\mathrm{x})=\frac{ \pm \mathrm{x} \wedge \mathrm{e}_{3}}{\left|\mathrm{x} \wedge \mathrm{e}_{3}\right|}
$$

then $E_{O}$ has an attained absolute minimum of the form (4.6) and in fact $(+)$ can be imposed on all of $S^{1} \times S^{1}$ so that the minimizer exhibits a single domain (note that $m$ defined by (4.6) is divergence-free). However, with unbent uniaxial crystals of sufficient size, we expect always to see fine structure throughout the crystal, as is observed.

\section{(b) Cubic case}

The preceding statement is untrue for cubic crystals where mm size single crystals of iron often exhibit the classic domain structure pictured in Figure 2a, if the faces of the crystal have been cut on (100) planes ( $c f$. Carey and Isaac [1966], Figure 101). In fact Figure 2a clearly represents a minimizer of $E_{0}$ since the field $m$ pictured there is divergence-free on $\mathbb{R}^{n}$ (and therefore has the corresponding potential $u=0$ on $\mathbb{R}^{n}$, and also $m$ assumes only the values $\pm m_{1}$ and $\pm m_{2}$. 


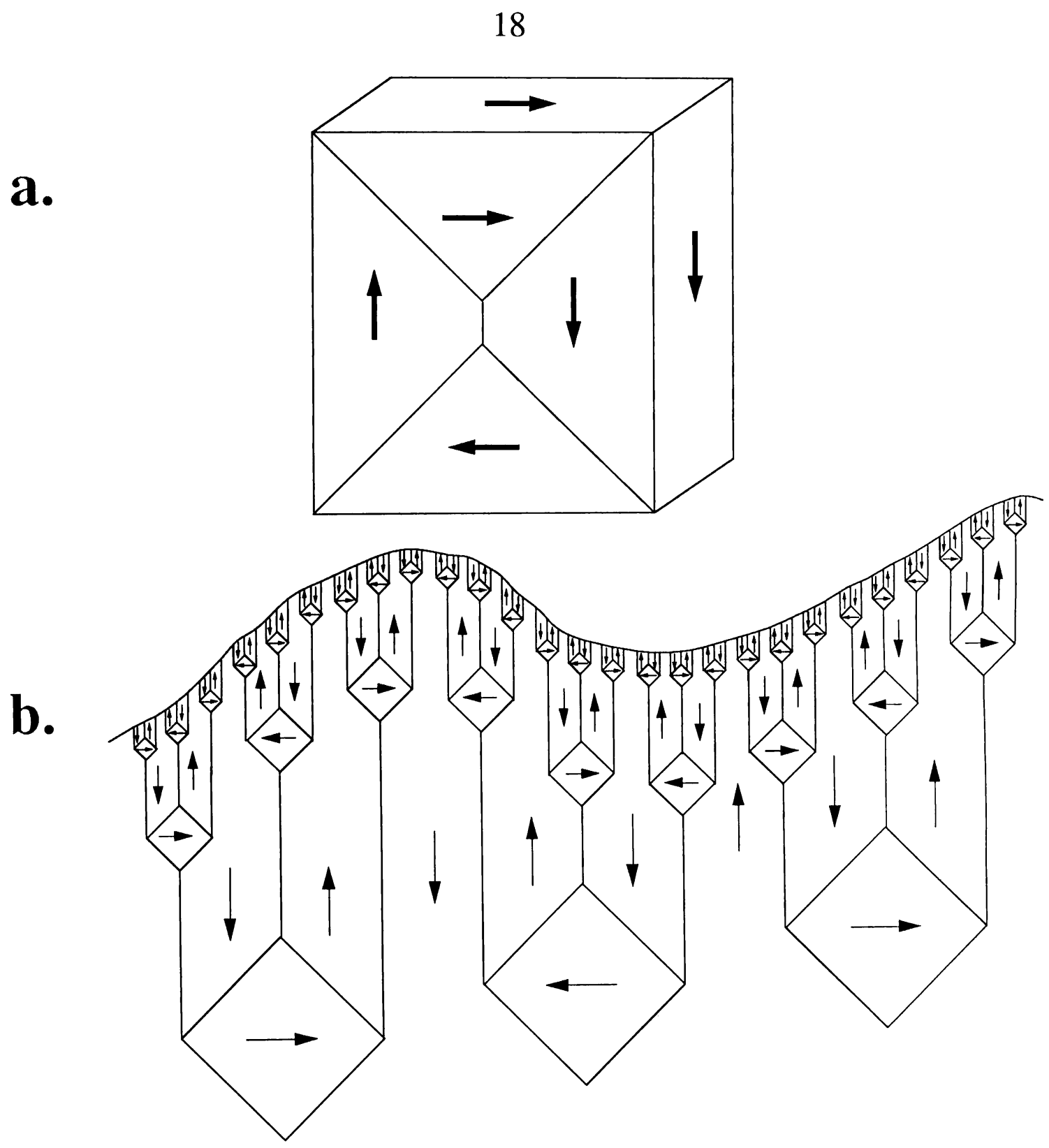

[001]

c.
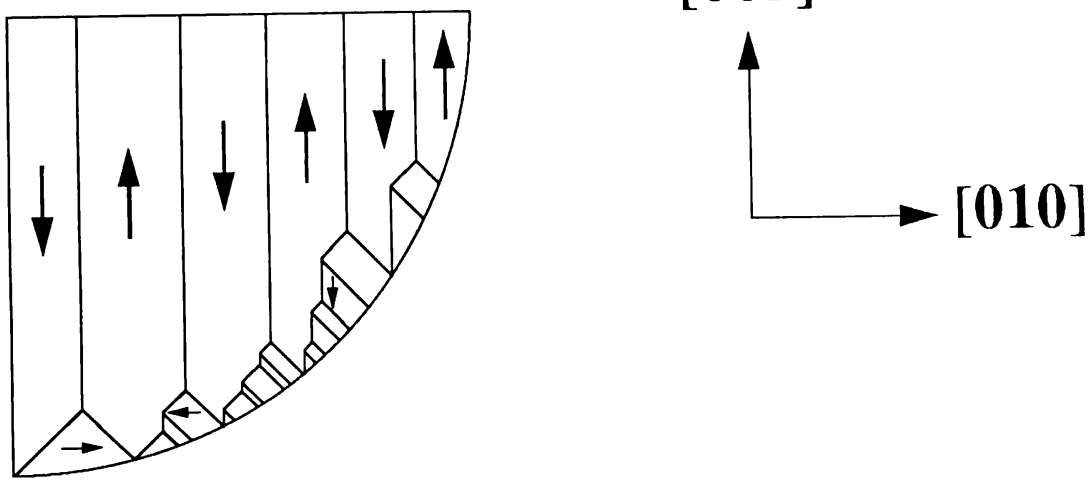

Figure 2. Minimizing domain structures in the cubic case. Figure $2 \mathrm{c}$ after Craik and Tebble [1965, Fig. 6.8a,b]. 
The question arises whether attainment of the minimum in the cubic case occurs only if $\partial \Omega$ exhibits (100) normals. To investigate this question we let $\mathrm{D}$, the prototype being the domain shown in Fig.2a, be some particular, open set in $\mathbb{R}^{\mathrm{n}}$ on which the minimum is attained by a field $\mathrm{m}_{\mathrm{o}} \in \mathrm{L}^{2}\left(\Omega ; \mathrm{S}^{\mathrm{n}-1}\right)$ :

$$
\mathrm{E}_{\mathrm{o}}^{\mathrm{D}}\left(\mathrm{m}_{\mathrm{o}}\right)=0
$$

where

$$
\mathrm{E}_{\mathrm{o}}^{\mathrm{D}}(\mathrm{m}):=\int_{\mathrm{D}} \varphi(\mathrm{m}(\mathrm{x})) \mathrm{dx}+\frac{1}{2} \int_{\mathbb{R}^{\mathrm{n}}}|\nabla \mathrm{u}|^{2} \mathrm{dx}
$$

The following shows that the minimum of $\mathrm{E}_{\mathrm{o}}^{\Omega}$ is attained for any open bounded $\Omega \subset \mathbb{R}^{n}$.

Theorem 4.2. Let $\Omega \subset \mathbb{R}^{\mathrm{n}}$ be open and bounded and let the open bounded set $\mathrm{D} \subset \mathbb{R}^{\mathrm{n}}$ have a smooth boundary. Suppose

$$
0=\inf \mathrm{E}_{\mathrm{o}}^{\mathrm{D}}=\mathrm{E}_{\mathrm{o}}^{\mathrm{D}}\left(\mathrm{m}_{\mathrm{o}}\right), \quad \mathrm{m}_{\mathrm{o}} \in \mathrm{L}^{2}\left(\mathrm{D} ; \mathrm{S}^{\mathrm{n}-1}\right)
$$

Then there is a function $\mathrm{m} \in \mathrm{L}^{2}\left(\Omega ; \mathrm{S}^{\mathrm{n}-1}\right)$ such that

$$
0=\inf E_{o}^{\Omega}=E_{o}^{\Omega}\left(m_{0}\right)
$$

Proof. By the Vitali Covering Theorem, there is a countable collection of disjoint closed sets of the form $a_{i}+\varepsilon_{i} \bar{D}$ such that

$$
\Omega=\cup\left(a_{i}+\varepsilon_{i} \bar{D}\right) \cup N
$$

where meas $\mathrm{N}=0$. Since $\mathrm{m}_{\mathrm{o}}$ is a minimizer on $\mathrm{D}$ 


$$
\int_{\mathbb{R}^{n}} \mathrm{~m}_{0} \chi_{\mathrm{D}} \cdot \nabla \zeta \mathrm{dx}=0 \text { for all } \zeta \in \mathrm{V}
$$

Let $m_{i} \in L^{2}\left(a_{i}+\varepsilon_{i} \bar{D} ; S^{n-1}\right)$ be defined by

$$
\mathrm{m}_{\mathrm{i}}(\mathrm{x}):=\mathrm{m}_{\mathrm{o}}\left(\frac{\mathrm{x}-\mathrm{a}_{\mathrm{i}}}{\varepsilon_{\mathrm{i}}}\right), \quad \mathrm{x} \in \mathrm{a}_{\mathrm{i}}+\varepsilon_{\mathrm{i}} \overline{\mathrm{D}}
$$

and let $m \in L^{2}\left(\Omega ; S^{n-1}\right)$ be defined by

$$
\mathrm{m}(\mathrm{x}):=\mathrm{m}_{\mathrm{i}}(\mathrm{x}), \quad \mathrm{x} \in \mathrm{a}_{\mathrm{i}}+\varepsilon_{\mathrm{i}} \overline{\mathrm{D}}
$$

We have for any $\zeta \in \mathrm{V}$

$$
\begin{aligned}
\int_{\mathbb{R}^{\mathrm{n}}} \mathrm{m}_{\mathrm{O}} \chi_{\mathrm{D}} \cdot \nabla \zeta \mathrm{dx} & =\int_{\Omega} \mathrm{m} \cdot \nabla \zeta \mathrm{dx} \\
& =\sum \int_{\mathrm{a}_{\mathrm{i}}+\varepsilon_{\mathrm{i}} \overline{\mathrm{D}}} \mathrm{m}_{\mathrm{i}} \cdot \nabla \zeta \mathrm{dx} \\
& =\sum \varepsilon_{\mathrm{i}}^{\mathrm{n}} \int_{\mathrm{D}} \mathrm{m}_{\mathrm{O}}(\mathrm{z}) \cdot \nabla \zeta\left(\mathrm{a}_{\mathrm{i}}+\varepsilon_{\mathrm{i}} \mathrm{z}\right) \mathrm{dz}
\end{aligned}
$$

where the series converges because the left hand side is finite. Since $\zeta_{\mathbf{i}}(\mathrm{z}):=\zeta\left(\mathrm{a}_{\mathbf{i}}+\varepsilon_{\mathrm{i}} \mathrm{z}\right)$ belongs to $\mathrm{V}$, up to an additive constant, each term in the series (4.13) vanishes. Therefore if $\mathrm{u}$ is the potential corresponding to $\mathrm{m}$ then, by (4.13), $\mathrm{u}(\mathrm{x})=0$ a.e. $\mathrm{x} \in \mathbb{R}^{\mathrm{n}}$, so $\mathrm{m}$ is a minimizer.

Since on domains with non-(100) boundaries there does not exist a piecewise smooth minimizer having a finite number of domains, the implication of Theorem 2 is that any minimizer will have the property that it will have a finer and finer domain structure at the boundary. This inference is made precise in Section 5. Various constructions are possible in addition to the one given by the Vitali Covering Theorem. For example, the construction shown in Figure $2 b$ also 
delivers a minimizer. Here it is important to observe that the average magnetization in Figure $2 \mathrm{~b}$ on a sequences of translates of $\partial \Omega$, which tend to $\partial \Omega$, goes to zero. Figure $2 \mathrm{c}$ shows an actual domain structure near the curved boundary of an iron crystal.

\section{Further Analysis of Domain Structures}

As illustrated by Figure 1, there is extensive lack of uniqueness associated with minimizing sequences for uniaxial materials. However, both of the minimizing sequences illustrated by Figure 1 have magnetization fields whose average is zero, in the sense of weak convergence. In this section we quantify this idea of uniqueness for any minimizing sequence in the uniaxial case using the notion of a Young measure.

This uniqueness is proved in the uniaxial case only. No such average behavior is expected for cubic magnets because of the dichotomy of scales illustrated by Figure 1 and 2a. However, whether or not a minimizer in the cubic case exhibits refinement at the boundary, we expect the average magnetization at the boundary to be tangential, and hence to satisfy in some sense the equation $\operatorname{div} \mathrm{m}=0$. We prove this below. It follows immediately from this fact that if $\partial \Omega$ is smooth and has a normal $v\left(x_{0}\right)$ at $x_{0} \in \partial \Omega$ not perpendicular to (100), (010) or (001), then any minimizer must exhibit refinement at $\mathrm{x}_{0}$.

First we make a few observations about the general situation. Let $\mathrm{m}^{\mathrm{k}} \in \mathrm{L}^{\infty}(\Omega),\left|\mathrm{m}^{\mathrm{k}}\right|=1$, be a minimizing sequence for $\mathrm{E}_{0}$, with potentials $\left(\mathrm{u}^{\mathrm{k}}\right)$. Thus

$$
\mathrm{E}_{0}\left(\mathrm{~m}^{\mathrm{k}}\right)=\int_{\Omega} \varphi\left(\mathrm{m}^{\mathrm{k}}\right) \mathrm{dx}+\frac{1}{2} \int_{\mathbb{R}^{\mathrm{n}}}\left|\nabla \mathrm{u}^{\mathrm{k}}\right|^{2} \mathrm{dx} \rightarrow 0 \text { as } \mathrm{k} \rightarrow \infty
$$

which tells us that both 


$$
\begin{aligned}
& \int_{\Omega} \varphi\left(\mathrm{m}^{\mathrm{k}}\right) \mathrm{dx} \rightarrow 0 \quad \text { and } \\
& \int_{\mathbb{R}^{\mathrm{n}}}\left|\nabla \mathrm{u}^{\mathrm{k}}\right|^{2} \mathrm{dx} \rightarrow 0 .
\end{aligned}
$$

Thus

$$
\begin{array}{ll}
\varphi\left(\mathrm{m}^{\mathrm{k}}\right) \rightarrow 0 & \text { in } \mathrm{L}^{1}(\Omega) \text { and } \\
\mathrm{u}^{\mathrm{k}} \rightarrow 0 & \text { in } \mathrm{V} \text { (in norm). }
\end{array}
$$

The boundedness of the $\left(\mathrm{m}^{\mathrm{k}}\right)$ means that there is a subsequence, not relabeled, and an $\overline{\mathrm{m}} \in$ $L^{\infty}\left(\mathbb{R}^{n}\right)$ such that

$$
\begin{aligned}
& \mathrm{m}^{\mathrm{k}} \rightarrow \overline{\mathrm{m}} \text { in } \mathrm{L}^{2}\left(\mathbb{R}^{\mathrm{n}}\right) \text { weakly and } \\
& \text { supp } \overline{\mathrm{m}} \subset \Omega .
\end{aligned}
$$

Since for each $\mathrm{k}$,

$$
\int_{\mathbb{R}^{\mathrm{n}}}\left(-\nabla \mathrm{u}^{\mathrm{k}}+\mathrm{m}^{\mathrm{k}}\right) \cdot \nabla \zeta \mathrm{dx}=0, \quad \zeta \in \mathrm{C}_{\mathrm{o}}^{\infty}\left(\mathbb{R}^{\mathrm{n}}\right),
$$

we obtain from (5.2) on passing to the limit that

$$
\int_{\mathbb{R}^{n}} \overline{\mathrm{m}} \cdot \nabla \zeta \mathrm{dx}=0, \quad \zeta \in \mathrm{C}_{\mathrm{o}}^{\infty}\left(\mathbb{R}^{\mathrm{n}}\right)
$$

or

$$
\operatorname{div} \overline{\mathrm{m}}=0 \text {. }
$$


The existence theorem for Young measures (e.g. Ball [1989], Young [1980]) states that there is a subsequence of $\left(\mathrm{m}^{\mathrm{k}}\right)$, not relabeled, and a family of probability measures $\left(\mu_{\mathrm{x}}\right)_{\mathrm{x} \in \Omega}$ such that for every $\psi \in C\left(\mathbb{R}^{n}\right)$,

$$
\begin{aligned}
& \psi\left(\mathrm{m}^{\mathrm{k}}\right) \rightarrow \bar{\psi} \quad \text { in } \mathrm{L}^{\infty}\left(\mathbb{R}^{\mathrm{n}}\right) \text { weakly, where } \\
& \bar{\psi}(\mathrm{x})=\int_{\mathbb{R}^{\mathrm{n}}} \psi(\mathrm{m}) \mathrm{d} \mu_{\mathrm{x}}(\mathrm{m}), \quad \mathrm{x} \in \Omega .
\end{aligned}
$$

Part of the conclusion of the theorem is that $\bar{\psi}$ is measurable. It follows from (5.5) that $\mu_{\mathrm{x}}$ has an interpretation as a local spatial average:

$$
\mu_{x}(E)=\lim _{r \rightarrow 0} \lim _{k \rightarrow \infty} \frac{1}{\left|B_{r}\right|}\left|\left\{z \in B(x, r): m^{k}(z) \in E\right\}\right|
$$

Also note that the limit magnetization given in (5.3) has the representation

$$
\overline{\mathrm{m}}(\mathrm{x})=\int_{\mathbb{R}^{\mathrm{n}}} \mathrm{m} \mathrm{d} \mu_{\mathrm{x}}(\mathrm{m})
$$

The limit anisotropy energy density is

$$
\bar{\varphi}(\mathrm{x})=\int_{\mathbb{R}^{\mathrm{n}}} \varphi(\mathrm{m}) \mathrm{d} \mu_{\mathrm{x}}(\mathrm{m})
$$

but since $0 \leq \varphi\left(\mathrm{m}^{\mathrm{k}}\right) \rightarrow 0, c f .(5.2)_{2}$,

$$
\int_{\mathbb{R}^{n}} \varphi(\mathrm{m}) \mathrm{d} \mu_{\mathrm{x}}(\mathrm{m})=0
$$

It follows immediately that

$$
\operatorname{supp} \mu_{x}=\{m: \varphi(m)=0\}=: K
$$


We now restrict attention to the uniaxial case, where $K=\left\{m_{1},-m_{1}\right\}$. In this case from (5.7)

$$
\begin{array}{ll}
\mu_{\mathrm{x}}=\lambda(\mathrm{x}) \delta_{\mathrm{m}_{1}}+(1-\lambda(\mathrm{x})) \delta_{-\mathrm{m}_{1}} & \text { in } \Omega, \text { and } \\
\overline{\mathrm{m}}(\mathrm{x})=(2 \lambda(\mathrm{x})-1) \mathrm{m}_{1} \chi_{\Omega} & \text { in } \Omega,
\end{array}
$$

where $0 \leq \lambda \leq 1$

On the other hand, by (5.4)

$$
0=\int_{\mathbb{R}^{\mathrm{n}}} \overline{\mathrm{m}} \cdot \nabla \zeta \mathrm{dx}=\int_{\mathbb{R}^{\mathrm{n}}}(2 \lambda(\mathrm{x})-1) \mathrm{m}_{1} \chi \Omega \cdot \nabla \zeta \mathrm{dx}, \quad \zeta \in \mathrm{C}_{\mathrm{o}}^{\infty}\left(\mathbb{R}^{\mathrm{n}}\right)
$$

From Lemma $4.6,2 \lambda(x)-1=0$ in $\Omega$, or $\lambda(x) \equiv \frac{1}{2}$ and $\bar{m}=0$. It is easily seen that these conclusions apply to the whole sequence.

We have proved the uniqueness theorem.

Proposition 5.1. Assume that $\varphi$ satisfies the uniaxial assumptions (2.5). Every minimizing sequence $\left(\mathrm{m}^{\mathrm{k}}\right)$ for $\mathrm{E}_{\mathrm{o}}$ satisfies

$$
\mathrm{m}^{\mathrm{k}} \rightarrow 0 \quad \text { in } \mathrm{L}^{\infty}\left(\mathbb{R}^{\mathrm{n}}\right) \text { weak* }
$$

and generates the Young measure

$$
\mu_{\mathrm{x}}=\frac{1}{2} \delta_{\mathrm{m}_{1}}+\frac{1}{2} \delta_{-\mathrm{m}_{1}}
$$

Now we turn to the cubic case. Let $\mathrm{m}$ be a minimizer of $\mathrm{E}_{\mathrm{o}}$, so in particular the potential corresponding to $\mathrm{m}$ is $\mathrm{u}=0$ and $\operatorname{div} \mathrm{m}=0$, or 


$$
\int_{\mathbb{R}^{n}} \mathrm{~m} \cdot \nabla \zeta \mathrm{dx}=0, \quad \zeta \in \mathrm{C}_{\mathrm{o}}^{\infty}\left(\mathbb{R}^{\mathrm{n}}\right)
$$

This is just a special case of (5.4). Let $\mathrm{a} \in \partial \Omega$ and $\zeta \in \mathrm{C}_{\mathrm{o}}^{\infty}(\mathrm{B}(\mathrm{a}, \mathrm{r}))$. We assume that $\partial \Omega$ is smooth near a. Integrating by parts, we get

$$
\begin{aligned}
0 & =\int_{\mathrm{B}(\mathrm{a}, \mathrm{r})} \mathrm{m} \cdot \nabla \zeta \mathrm{dx} \\
& =\int_{\mathrm{B}(\mathrm{a}, \mathrm{r}) \cap \Omega} \mathrm{m} \cdot \nabla \zeta \mathrm{dx} \\
& =\int_{\partial \Omega \cap \mathrm{B}(\mathrm{a}, \mathrm{r})} \mathrm{m} \cdot v \zeta \mathrm{dS}
\end{aligned}
$$

Here, $\left.\mathrm{m}\right|_{\partial \Omega}$ is defined by routine mollification, i.e.,

$$
\mathrm{m}_{\partial \Omega}=\lim _{\varepsilon \rightarrow 0} \mathrm{~m}_{\mathcal{E}} \quad \text { in } \mathrm{L}^{\infty}(\partial \Omega) \text { weak* }
$$

where

$$
m_{\varepsilon}(x)=\int_{\mathbb{R}^{n}} \alpha_{\varepsilon}\left(x-x^{\prime}\right) m\left(x^{\prime}\right) d x^{\prime}
$$

and $\left(\alpha_{\varepsilon}\right)$ is a sequence of mollifiers. Formula (5.8) may be seen directly from the fact that

$$
\operatorname{div} \mathrm{m}_{\varepsilon}=0
$$

Indeed,

$$
\begin{aligned}
0 & =\int_{\mathrm{B}(\mathrm{a}, \mathrm{r}) \cap \Omega} \operatorname{div} \mathrm{m}_{\varepsilon} \zeta \mathrm{dx} \\
& =-\int_{\mathrm{B}(\mathrm{a}, \mathrm{r}) \cap \Omega} \mathrm{m}_{\mathcal{\varepsilon}} \cdot \nabla \zeta \mathrm{dx}+\int_{\partial \Omega \cap \mathrm{B}(\mathrm{a}, \mathrm{r})} \mathrm{m}_{\mathcal{\varepsilon}} \cdot v \zeta \mathrm{dx} .
\end{aligned}
$$

Now let $\varepsilon \rightarrow 0$ so 


$$
0=-\int_{\mathrm{B}(\mathrm{a}, \mathrm{r}) \cap \Omega} \mathrm{m} \cdot \nabla \zeta \mathrm{dx}+\int_{\partial \Omega \cap \mathrm{B}(\mathrm{a}, \mathrm{r})} \mathrm{m} \cdot v \zeta \mathrm{dx} .
$$

But $\mathrm{m}=\mathrm{m} \chi \Omega$, whence

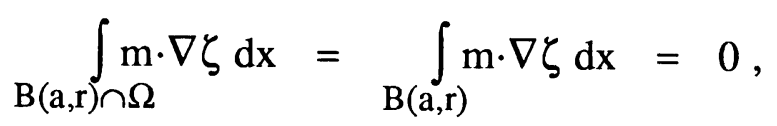

which yields (5.8). From (5.8), we obtain that

$$
\mathrm{m}_{\partial \Omega} \cdot v=0 \quad \text { on } \partial \Omega
$$

Returning to the issue of boundary refinement, suppose that $\partial \Omega$ is smooth near $a \in \partial \Omega$ and that for every sufficiently small $\varepsilon>0, \mathrm{~B}(\mathrm{a}, \varepsilon) \cap \Omega$ contains a single domain:

$$
\mathrm{m}(\mathrm{x})=+\mathrm{m}_{1} \text {, say, for a.e. } \mathrm{x} \in \mathrm{B}(\mathrm{a}, \varepsilon) \cap \Omega \text {. }
$$

Assume for simplicity that $n=3$. It follows from (5.9)-(5.11) that $v$ is perpendicular to $m_{1}$. Conversely, if $v$ at $a \in \partial \Omega$ is such that $v \cdot \mathrm{m}_{\mathrm{i}} \neq 0, \mathrm{i}=1,2,3$, then there must be a sequence $\varepsilon_{\mathrm{k}}$ $\rightarrow 0$ such that each set $\mathrm{B}\left(\mathrm{a}, \varepsilon_{\mathrm{k}}\right)$ contains at least two sets of positive measures on which $\mathrm{m}$ takes on different values in $\left\{ \pm m_{1}, \pm m_{2}, \pm m_{3}\right\}$. Hence, boundary refinement is necessary in cubic materials if the boundary normal is not perpendicular to one of the directions $(100),(010),(001)$.

This result does not explain the refinement observed in Figure 2c, since the normal in that case is perpendicular to (100). This is explained by the following argument. The disc shown in figure $2 \mathrm{c}$ is a cylinder with the top normal to $\mathrm{m}_{1}=(100)$. The domains viewed there are on the top. Suppose that $a \in \partial \Omega$ lies on a corner of the cylinder and the normal $v(a)$ to the lateral boundary of the cylinder at $a$ is $v(a)=\alpha m_{1}+\beta m_{2}$ with both $\alpha \neq 0$ and $\beta \neq 0$. Furthermore, suppose there is a single domain $\mathrm{m}(\mathrm{x})=+\mathrm{m}_{\mathrm{k}}, \mathrm{k} \in\{1,2,3\}$ in $\mathrm{B}(\mathrm{a}, \varepsilon) \cap \Omega$. Then by (5.11), 
$\mathrm{m}_{\mathrm{k}} \cdot \mathrm{m}_{3}=0$ and $\mathrm{v}(\mathrm{a}) \cdot \mathrm{m}_{\mathrm{k}}=0$. This is a contradiction, in other words, there is no single domain in a ball of any size. This leads to boundary refinement.

\section{Bound on the Minimum Energy via a Lagrangian Formulation}

In preparation for the discussion of the effect of an applied field, we find a lower bound on the infimum of the total energy. The presence of a divergence-free applied field $h_{0} \in L^{2}\left(\mathbb{R}^{n} ; \mathbb{R}^{n}\right)$ contributes a term $-m \cdot h_{0}$ to the local part of the energy resulting in a total energy

$$
\mathrm{E}\left(\mathrm{m} ; \mathrm{h}_{\mathrm{o}}\right)=\int_{\Omega}\left(\varphi(\mathrm{m})-\mathrm{m} \cdot \mathrm{h}_{\mathrm{o}}\right) \mathrm{dx}+\frac{1}{2} \int_{\mathbb{R}^{\mathrm{n}}}|\nabla \mathrm{u}|^{2} \mathrm{dx}
$$

subject to

$$
\operatorname{div}\left(-\nabla \mathrm{u}+\mathrm{m} \chi_{\Omega}\right)=0 \quad \text { in } \mathrm{V}
$$

The field $h_{0}$ is interpreted as the field that would be present were the ferromagnetic material absent, $c f$. Brown [1962]. Using (6.2), we can write

$$
\mathrm{E}\left(\mathrm{m}, \mathrm{u} ; \mathrm{h}_{\mathrm{O}}\right)=\int_{\Omega}\left(\varphi(\mathrm{m})-\mathrm{m} \cdot\left(\mathrm{h}_{\mathrm{O}}-\nabla \mathrm{u}\right)\right) \mathrm{dx}-\frac{1}{2} \int_{\mathbb{R}^{\mathrm{n}}}|\nabla \mathrm{u}|^{2} \mathrm{dx}
$$

Regarding $\mathrm{h}_{\mathrm{o}}$ as fixed, we introduce a "Lagrangian,"

$$
\begin{aligned}
\mathrm{L}(\mathrm{m}, \mathrm{u}) & =\frac{1}{2} \int_{\mathbb{R}^{\mathrm{n}}}|\nabla \mathrm{u}|^{2} \mathrm{dx}+\int_{\Omega}\left\{\mathrm{m} \cdot\left(\mathrm{h}_{\mathrm{O}}-\nabla \mathrm{u}\right)-\varphi(\mathrm{m})\right\} \mathrm{dx} \\
& =-\mathrm{E}\left(\mathrm{m}, \mathrm{u} ; \mathrm{h}_{\mathrm{O}}\right)
\end{aligned}
$$

Let us regard $\mathrm{L}(\mathrm{m}, \mathrm{u})$ as a mapping from $\mathbb{K} \times \mathrm{V} \rightarrow \mathbb{R}$, where $\mathrm{V}$ is defined by (3.2) and

$$
\mathbb{K}=\left\{\mathrm{m} \in \mathrm{L}^{\infty}\left(\Omega ; \mathbb{R}^{\mathrm{n}}\right):|\mathrm{m}|=1\right\}
$$


So, in (6.4) we ignore the equation (6.2). The reason for this is that it may be directly incorporated into the variational principle by observing that

$$
-\mathrm{P}^{*}=\inf _{\substack{\mathrm{m} \in \mathbb{K} \\ \text { u satisfies }(6.2)}} \mathrm{E}(\mathrm{m})=-\sup _{\mathbb{K}} \inf _{\mathrm{V}} \mathrm{L}(\mathrm{m}, \mathrm{u})
$$

Formally, if we compute the first variation $\delta \mathrm{L}(\mathrm{m}, \mathrm{u})$, we get (6.2) as well as the Euler equation of (6.1). Indeed, $-\mathrm{L}(\mathrm{m}, \mathrm{u})$ is often regarded as the Helmholtz free energy of the system. The proof of (6.5) is an immediate consequence of our proof of Lemma 3.1.

Let

$$
P=\inf _{V} \sup _{\mathbb{K}} L(m, u)
$$

By an elementary computation (cf., e.g., Ekeland and Temam [1976] or Moreau [1964]),

$$
\mathrm{P}^{*} \leq \mathrm{P}
$$

Thus (-P) provides a lower bound for the energy attained by the system. The advantage of (6.6) is that there is always a pair $(\mathrm{m}, \mathrm{u})$ which attains (6.6), and this gives a sharp bound in some cases.

Let us calculate $\mathrm{P}$ from a variational problem. Define

$$
\psi(\xi)=\sup _{|\mathrm{m}|=1}(\mathrm{~m} \cdot \xi-\varphi(\mathrm{m}))
$$

Then for each $u \in V$, we set

$$
\mathrm{I}(\mathrm{u})=\sup _{\mathbb{K}} \mathrm{L}(\mathrm{m}, \mathrm{u})=\frac{1}{2} \int_{\mathbb{R}^{\mathrm{n}}}|\nabla \mathrm{u}|^{2} \mathrm{dx}+\int_{\Omega} \psi\left(\mathrm{h}_{\mathrm{O}}-\nabla \mathrm{u}\right) \mathrm{dx}
$$

Assuming that $\varphi$ is even and $\inf \varphi=0$, we easily deduce that $|\mathrm{m}|=1$ 
(i) $\psi$ is convex and continuous,

(ii) $\psi(-\xi)=\psi(\xi)$, and

(iii) $\inf \psi=\psi(0)=\inf _{|\mathrm{m}|} \mid=1 \varphi=0$.

To check (iii), note that a convex even function assumes its minimum at the origin. Thus $I$ is convex and coercive on $V$. Hence there is a (unique) $\tilde{u} \in V$ such that

$$
\mathrm{I}(\tilde{\mathrm{u}})=\inf _{\mathrm{V}} \mathrm{I}(\mathrm{u})=\mathrm{P}
$$

As a means of estimating $\mathrm{P}$, let us note a special case. Let

$$
\mathrm{V}_{\mathrm{o}}=\left\{\mathrm{u} \in \mathrm{V}: \nabla \mathrm{u}=\mathrm{h}_{\mathrm{o}} \text { in } \Omega\right\} .
$$

If the closed, convex set $\mathrm{V}_{\mathrm{o}}$ is not empty, there is a unique $\tilde{\mathrm{u}} \in \mathrm{V}_{\mathrm{o}}$ such that

$$
\int_{\mathbb{R}^{\mathrm{n}}}|\nabla \tilde{\mathrm{u}}|^{2} \mathrm{dx}=\inf _{\mathrm{v}_{\mathrm{o}}} \int_{\mathbb{R}^{\mathrm{n}}}|\nabla \mathrm{u}|^{2} \mathrm{dx},
$$

by Stampacchia's Theorem [1964]. Hence $\psi\left(\mathrm{h}_{\mathrm{o}}-\nabla \tilde{\mathrm{u}}\right)=0$ on $\Omega$ and, by (6.9)

$$
P \leq I(\tilde{\mathrm{u}})=\frac{1}{2} \int_{\mathbb{R}^{\mathrm{n}}}|\nabla \tilde{\mathrm{u}}|^{2} \mathrm{dx} .
$$

When $h_{0} \neq 0,(6.11)$ will provide an optimal bound only for special domains in $\Omega$. Of course, if $\mathrm{h}_{\mathrm{O}}=0$, then $\tilde{\mathrm{u}}=0$ and $\mathrm{P}=0$ as well. In Section 3 we have shown that $\mathrm{P}^{*}=0$ in this case, so the bound given by this formulation is optimal.

Another simple case, when $\Omega$ is an ellipsoid and $h_{0}=$ const., is treated in Section 7.

The numbers $\mathrm{P}$ and $\mathrm{P}^{*}$ would be equal were it possible to interchange the "inf" and "sup" in (6.5) or (6.6). For general fields $h_{0}$ this may not be possible. First of all, $\mathrm{P}^{*}$ is not 
attained in general, which renders less tractable the computations. A true saddle point does not exist in this case. Moreover, $\psi$ is not the dual function $\varphi^{*}$ of $\varphi$ owing to the constraint that $|\mathrm{m}|$ $=1$, which refers to the assumption of magnetic saturation. Consonant with this, the set $\mathbb{K}$ is not convex. We refer to Ekeland and Temam [1976] for a general discussion.

The idea of the Lagrangian formulation may be recast in an extended setting. Let $\mathrm{N} \subset \mathrm{S}^{\mathrm{n}-1}$ and

$$
\mathbb{N}=\left\{m \in L^{\infty}\left(\Omega ; S^{n-1}\right): m(x) \in N\right\}
$$

and introduce

$$
\psi_{\mathrm{N}}(\xi)=\sup _{\mathrm{N}}(\xi \cdot \mathrm{m}-\varphi(\mathrm{m}))
$$

which is convex with at most linear growth. For a fixed applied field $h_{0}$, set

$$
\mathrm{P}^{*}(\mathbb{N})=\sup _{\mathbb{N}} \inf _{\mathrm{V}} \mathrm{L}(\mathrm{m}, \mathrm{u}) \text { and } \mathrm{P}(\mathbb{N})=\inf _{\mathrm{V}} \sup _{\mathbb{N}} \mathrm{L}(\mathrm{m}, \mathrm{u})
$$

As before, $\mathrm{P}^{*}(\mathbb{N}) \leq \mathrm{P}(\mathbb{N})$. The convexity of $\psi_{\mathrm{N}}$ ensures that there is always a $\mathrm{u}_{\mathrm{N}} \in \mathrm{V}$ such that

$$
\mathrm{P}(\mathbb{N})=\frac{1}{2} \int_{\mathbb{R}^{\mathrm{n}}}\left|\nabla \mathrm{u}_{\mathrm{N}}\right|^{2} \mathrm{dx}+\int_{\Omega} \psi_{\mathrm{N}}\left(\mathrm{h}_{\mathrm{O}}-\nabla \mathrm{u}_{\mathrm{N}}\right) \mathrm{dx}
$$

We shall use this extended setting to interpret the notion of metastable solutions of our problem, and in particular the coercivity paradox, in the Section 8 . 


\section{Effect of a Constant Applied Field}

In the preceding section we showed that the minimum energy $\mathrm{P}^{*}$ in the presence of an applied field satisfies

$$
-\mathrm{P}^{*}=\inf _{\mathrm{m} \in \mathbb{K}} \mathrm{E}\left(\mathrm{m} ; \mathrm{h}_{\mathrm{o}}\right) \geq-\mathrm{P} \geq-\frac{1}{2} \int_{\mathbb{R}^{\mathrm{n}^{-}}}|\nabla \tilde{\mathrm{u}}|^{2} \mathrm{dx} .
$$
u satisfies (6.2)

provided that $\tilde{\mathrm{u}} \in \mathrm{V}_{\mathrm{h}_{\mathrm{o}}}$ minimizes the field energy

$$
\int_{\mathbb{R}^{n}}|\nabla \tilde{u}|^{2} \mathrm{dx}=\inf _{\mathrm{V}_{\mathrm{h}_{\mathrm{o}}}} \int_{\mathbb{R}^{\mathrm{n}}}|\nabla \mathrm{u}|^{2} \mathrm{dx}
$$

among other fields in $\mathrm{V}_{\mathrm{h}_{\mathrm{o}}}$.

In this section we consider the case $h_{o}=$ const. on $\Omega$, and we assume $n=3$.

It is well-known from potential theory that if $\Omega$ is an ellipsoid, the infimum in (7.2) is attained by a function $\tilde{\mathrm{u}}$ that is exactly the magnetic field of a uniformly magnetized ellipsoid with magnetization $\overline{\mathrm{m}}=\mathrm{D}^{-1} \mathrm{~h}_{\mathrm{o}}$. Here $\mathrm{D}=\mathrm{D}^{\mathrm{T}}$ is coaxial with the principal axes of $\Omega$ and its positive eigenvalues are the so-called demagnetizing factors (cf. Osborn [1945] or Stoner [1945]). The function $\tilde{\mathrm{u}}$ is obtained by solving

$$
\tilde{\mathrm{u}} \in \mathrm{V}: \int_{\mathbb{R}^{3}}\left(-\nabla \tilde{\mathrm{u}}+\overline{\mathrm{m}} \chi_{\Omega}\right) \cdot \nabla \zeta \mathrm{dx}=0 \quad \text { for all } \zeta \in \mathrm{V} .
$$

We now show that equality holds throughout (7.1) in the special case where $\Omega$ is an ellipsoid with demagnetizing matrix $\mathrm{D}$, and $\mathrm{h}_{\mathrm{o}}$ satisfies

$$
\begin{aligned}
& h_{\mathrm{o}} \| \mathrm{Dm}_{1}, \\
& \left|\mathrm{~h}_{\mathrm{o}}\right| \leq\left|D \mathrm{D}_{1}\right| .
\end{aligned}
$$


Recall that $m_{1}$ minimizes the anisotropy energy $\varphi$. Assume (7.3) and for $\lambda \in[0,1]$, let the 1periodic function $\theta_{\lambda}$ be given by

$$
\theta_{\lambda}(t)=\left\{\begin{array}{cc}
1 & t \in[0, \lambda) \\
-1 & t \in[\lambda, 1)
\end{array}\right.
$$

Choose $\mathrm{p} \in \mathbb{R}^{3}$ with $\mathrm{p} \cdot \mathrm{m}_{1}=0$ and let

$$
\mathrm{m}_{\lambda}^{\mathrm{k}}(\mathrm{x})=\left\{\begin{array}{c}
\mathrm{m}_{1} \theta_{\lambda}(\mathrm{kx} \cdot \mathrm{p}), \mathrm{x} \in \Omega, \\
0, \mathrm{x} \in \mathbb{R}^{3}-\Omega .
\end{array}\right.
$$

Then for each fixed $\lambda \in[0,1]$,

$$
\mathrm{m}_{\lambda}^{\mathrm{k}}(\mathrm{x}) \rightarrow \overline{\mathrm{m}}_{\lambda}:=(2 \lambda-1) \chi_{\Omega} \mathrm{m}_{1} \quad \text { weakly in } \mathrm{L}^{2},
$$

so by the theory presented in Section 3 (cf. equation (3.11)),

$$
\begin{aligned}
& \frac{1}{2} \int_{\Omega} \mathrm{m}_{\lambda}^{\mathrm{k}} \cdot \nabla \mathrm{u}_{\lambda}^{\mathrm{k}} \mathrm{dx} \rightarrow \frac{1}{2} \int_{\Omega} \overline{\mathrm{m}} \cdot \nabla \overline{\mathrm{u}} \lambda \mathrm{dx}, \\
& \int_{\Omega} \mathrm{m}^{\mathrm{k}} \cdot \mathrm{h}_{\mathrm{o}} \mathrm{dx} \rightarrow \int_{\Omega} \overline{\mathrm{m}} \cdot \mathrm{h}_{\mathrm{o}} \mathrm{dx},
\end{aligned}
$$

where $u_{\lambda}^{k}$ is the potential for $m_{\lambda}^{k}$ and

$$
\mathrm{u}_{\lambda}^{\mathrm{k}} \rightarrow \overline{\mathrm{u}} \lambda \text { weakly in } \mathrm{V} \text {. }
$$

It follows from (7.4) and (7.6) that $\overline{\mathrm{u}} \lambda$ is the potential corresponding to the constant magnetization $\overline{\mathrm{m}} \lambda$ on $\Omega$. Thus, we can ensure that $\overline{\mathrm{u}} \lambda$ will be the minimizer of the problem (7.2) if we choose $\lambda \in[0,1]$ such that

$$
\operatorname{Dim}_{\lambda}=(2 \lambda-1) \mathrm{Dm}_{1}=\mathrm{h}_{\mathrm{o}},
$$


in which case $\nabla \mathrm{u}_{\lambda}=\mathrm{h}_{\mathrm{O}}$ on $\Omega$. By the assumption (7.3) it is always possible to choose such a $\lambda$.

We now evaluate directly the energy of the sequence $m_{\lambda}^{k}$. By using $(7.5)_{1,2}$ in the expression (6.3), we get

$$
-\frac{1}{2} \int_{\mathbb{R}^{3}}|\nabla \overline{\mathrm{u}}|^{2} \mathrm{dx} \leq-\mathrm{P}^{*}
$$

We have arranged that $\overline{\mathrm{u}}_{\lambda}$ achieves the minimum in (7.2) so by (7.1) we also have

$$
-\mathrm{P}^{*} \geq-\frac{1}{2} \int_{\mathbb{R}^{3}} \mid \nabla \overline{\mathrm{u}} \lambda^{2} \mathrm{dx}
$$

Thus, we have calculated $\mathrm{P}^{*}$ and clearly $\left(\mathrm{m}_{\lambda}^{\mathrm{k}}\right)$ provides a minimizing sequence for the energy.

In summary, if $\Omega$ is an ellipsoid with demagnetizing matrix $\mathrm{D}$ subject to a constant applied field $h_{0}$ satisfying (7.3), then the infimum of the total energy is given by

$$
-\frac{1}{2} \int_{\mathbb{R}^{3}} \mid \nabla \overline{\mathrm{u}} \lambda^{2} \mathrm{dx}
$$

where $\overline{\mathrm{u}}_{\lambda}$ is the potential corresponding to the constant magnetization $\overline{\mathrm{m}}_{\lambda}=(2 \lambda-1) \mathrm{m}_{1}$ and $\lambda$ $\in[0,1]$ is chosen so that $(2 \lambda-1) m_{1}=D^{-1} h_{0}$. Minimizing sequences for the total energy are given by uniformly layered microstructures with magnetization $\mathrm{m}_{1},-\mathrm{m}_{1}, \mathrm{~m}_{1},-\mathrm{m}_{1}$ and having the volume fraction $\lambda$. 


\section{Remarks on the Calculations of Brown and Lifshitz}

Despite the general attractiveness of the philosophy of micromagnetics, it has met with limited success. A typical attitude toward micromagnetics by experimentalists is the view of Carey and Isaac [1966]:

Since, in principle, the minimization [of the total free energy] is a straightforward problem, micromagnetics needs to postulate no domains or walls; if these are real the theory should predict them. While this approach is undoubtedly rigorous, it seems clear that the application, at this stage, has most value in the study of particles insufficiently large to support domain walls. In bulk specimens, conventional domain theory, despite its shortcomings, has the advantage of pictorial guidance from experimental domain observations and in most cases has proved successful in accounting for the results obtained.

It appears that this hesitancy toward micromagnetics arises from an interesting calculation of Brown [1962, p. 125-133; 1963, p. 66-72]. Brown's calculation concerns the metastability of the single domain state under constant applied field. The idea of the calculation is the following. For an appropriately oriented ellipsoid $\Omega$ subject to a large, suitably directed, constant applied field $h_{0}$, we expect the single domain state $m=m_{1}$ to be an absolute minimizer of the energy. (This follows from a calculation similar to the one we presented in Sections 6 and 7, as explained below). Brown considers the family of fields $h_{0}=D m_{1}+\tau m_{1}$ with $\tau$ decreasing from $+\infty$ toward $-\infty$. We expect that for sufficiently small values of $\tau$, the single domain state ceases to be metastable. Brown calculates sufficient conditions on $\tau$ that the magnetization $m(x)=m_{1}, x \in$ $\Omega$, makes the second variation of the energy positive definite at $\mathrm{m}_{1}$.

We can easily reproduce Brown's calculation* of metastability using our Lagrangian formulation of Section 6. Let $\mathrm{m}_{\mathrm{o}}$ be a point of local convexity of the anisotropy energy corresponding to the conjugate variable $\xi_{0}$, i.e. assume there is an $\varepsilon>0$ such that

* Actually, this is a slightly improved version of his calculation in that we prove $\mathrm{m}_{\mathrm{o}}$ is a minimizer relative to other fields $m$ satisfying sup $\left|m-m_{0}\right|<\varepsilon$, i.e. our argument does not rely on the use of the second variation. 


$$
\varphi(m)-\varphi\left(m_{0}\right)-\left(m-m_{0}\right) \cdot \xi_{0} \geq 0 \text { for all } m \in \mathbb{K}_{\varepsilon}
$$

where

$$
\mathbb{K}_{\mathcal{\varepsilon}}=\left\{\mathrm{m} \in \mathrm{L}^{\infty}:|\mathrm{m}|=1 \text { and }\left|\mathrm{m}-\mathrm{m}_{0}\right|<\varepsilon\right\}
$$

In terms of the extended setting described at the end of Section 6 , we are choosing $\mathbb{N}=\mathbb{K}_{\varepsilon}$. Assume also that $\Omega$ is an ellipsoid with demagnetizing matrix $\mathrm{D}(c f$. Section 7$)$ and that

$$
\mathrm{h}_{\mathrm{o}}=\xi_{\mathrm{o}}+\mathrm{Dm} \mathrm{m}_{\mathrm{o}}
$$

The argument of Section 6 leading to (6.9) does not depend on the precise form of the compact set $\mathbb{K}$, so in particular we can repeat the argument with $\mathbb{K}$ replaced by $\mathbb{K}_{\mathcal{\varepsilon}}$ and obtain (6.9), except that $\psi$ is now replaced by $\psi_{\varepsilon}$, where

$$
\psi_{\varepsilon}(\xi)=\sup _{\mathrm{K}_{\varepsilon}}(\mathrm{m} \cdot \xi-\varphi(\mathrm{m})), \quad \mathrm{K}_{\varepsilon}=\left\{\mathrm{m} \in \mathrm{S}^{3}:\left|\mathrm{m}-\mathrm{m}_{\mathrm{O}}\right|<\varepsilon\right\}
$$

In the present calculation, $\psi_{\varepsilon}$ remains convex and continuous but is no longer even and is not generally minimized at the origin. Since $\mathrm{m}_{\mathrm{o}}$ satisfies $(8.1)$, we have

$$
\psi_{\varepsilon}\left(\xi_{0}\right)=\xi_{o} \cdot m_{0}-\varphi\left(m_{o}\right)
$$

Let $u_{0} \in V$ be the field associated with $m_{0}$ :

$$
\int_{\mathbb{R}^{\mathrm{n}}}\left(-\nabla \mathrm{u}_{\mathrm{o}}+\mathrm{m}_{\mathrm{o}} \chi_{\Omega}\right) \cdot \nabla \zeta \mathrm{dx}=0
$$

By the results from potential theory mentioned in Section $7, \nabla \mathrm{u}_{\mathrm{o}}=\mathrm{Dm}_{\mathrm{o}}$ on $\Omega$ and $\mathrm{u}_{\mathrm{o}}$ satisfies 


$$
\int_{\mathbb{R}^{n}}\left|\nabla u_{0}\right|^{2} \mathrm{dx}=\inf _{\mathrm{V}_{\mathrm{Dm}_{\mathrm{o}}}} \int_{\mathbb{R}^{\mathrm{n}}}|\nabla \mathrm{u}|^{2} \mathrm{dx}
$$

Thus, using (8.2), (8.3) and (8.4), we get from (6.9),

$$
\mathrm{P}=\inf _{\mathrm{V}} \mathrm{I}(\mathrm{u}) \leq \mathrm{I}\left(\mathrm{u}_{\mathrm{o}}\right)=\frac{1}{2} \int_{\mathbb{R}^{\mathrm{n}}}\left|\nabla \mathrm{u}_{\mathrm{o}}\right|^{2} \mathrm{dx}+\int_{\Omega} \psi\left(\xi_{\mathrm{o}}\right) \mathrm{dx} .
$$

Since $-\mathrm{P}$ is a lower bound for the energy ( $c f .(6.5)$ and (6.9) specialized to $\mathbb{K}=\mathbb{K}_{\mathcal{E}}$ ) we have proved that

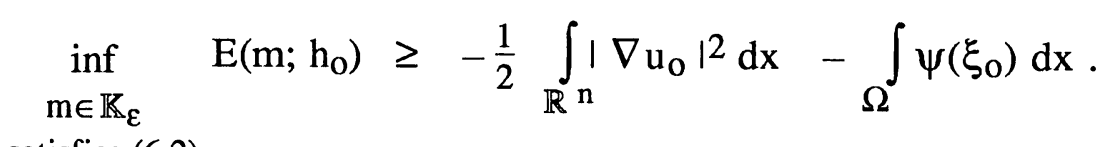
u satisfies (6.2)

But, in fact, this lower bound is attained by $\mathrm{m}_{0} \in \mathbb{K}_{\mathcal{E}}$ because

$$
\begin{aligned}
& \mathrm{E}\left(\mathrm{m}_{\mathrm{o}} ; \mathrm{h}_{\mathrm{o}}\right)=\int_{\Omega}\left(\varphi\left(\mathrm{m}_{\mathrm{o}}\right)-\mathrm{m}_{\mathrm{o}} \cdot \mathrm{h}_{\mathrm{o}}\right) \mathrm{dx}+\frac{1}{2} \int_{\mathbb{R}^{\mathrm{n}}}\left|\nabla \mathrm{u}_{\mathrm{o}}\right|^{2} \mathrm{dx} \\
& =\int_{\Omega}\left(\varphi\left(\mathrm{m}_{\mathrm{o}}\right)-\mathrm{m}_{\mathrm{o}} \cdot \mathrm{h}_{\mathrm{o}}+\mathrm{m}_{\mathrm{o}} \cdot \nabla \mathrm{u}_{\mathrm{o}}\right) \mathrm{dx}-\frac{1}{2} \int_{\mathbb{R}^{\mathrm{n}}}\left|\nabla \mathrm{u}_{\mathrm{o}}\right|^{2} \mathrm{dx} \\
& =-\int_{\Omega} \psi\left(\xi_{\mathrm{o}}\right) \mathrm{dx}-\frac{1}{2} \int_{\mathbb{R}^{\mathrm{n}}}\left|\nabla \mathrm{u}_{\mathrm{o}}\right|^{2} \mathrm{dx} .
\end{aligned}
$$

In summary, if $\mathrm{m}_{\mathrm{O}}$ satisfies $(8.1)$ and $\mathrm{h}_{\mathrm{O}}$ satisfies (8.2), then

$$
\mathrm{E}\left(\mathrm{m}_{\mathrm{o}} ; \mathrm{h}_{\mathrm{o}}\right)=\inf _{\substack{\mathrm{m} \in \mathbb{K}_{\varepsilon} \\ \text { u satisfies }(6.2)}} \mathrm{E}\left(\mathrm{m} ; \mathrm{h}_{\mathrm{o}}\right)
$$

This is essentially Brown's result on metastability. We note that this result remains true when exchange energy is included, since the exchange energy itself is minimized at the constant state $\mathrm{m}(\mathrm{x})=\mathrm{m}_{\mathrm{o}}, \mathrm{x} \in \Omega$. 
The metastability result (8.5) yields an interesting prediction which in turn leads to what Brown terms the "coercivity paradox." To obtain this prediction, we first calculate the set of values of $\xi_{0}$ for which $m_{0}$ is a point of local convexity. (This set determines a set of applied fields $h_{0}$ such that $m_{0}$ is metastable, by (8.2)). To correspond to Brown's treatment, we assume that

(i) $\varphi$ is even,

(ii) $\min _{\substack{\mathrm{Im} I=1 \\ \mathrm{~m} \cdot \mathrm{m}_{\mathrm{o}}=0}}\left[\mathrm{~m} \cdot \frac{\partial^{2} \varphi\left(\mathrm{m}_{\mathrm{o}}\right)}{\partial \mathrm{m}^{2}} \mathrm{~m}\right]=\kappa_{1}>0$.

It is then straightforward to determine the set of values of $\xi_{0}$ such that $m_{0}$ is a point of local convexity corresponding to $\xi_{\mathrm{o}}$. A convenient method of doing the calculation is by writing $\mathrm{m}=$ $\mathrm{R}(\mathrm{t}) \mathrm{m}_{\mathrm{O}}$ with $\mathrm{R}(0)=1, \dot{\mathrm{R}}(0)=\mathrm{W}=-\mathrm{W}^{\mathrm{T}}, \ddot{\mathrm{R}}(0)=\mathrm{W}^{2}+\dot{\mathrm{W}}, \dot{\mathrm{W}}^{\mathrm{T}}=-\dot{\mathrm{W}}$. It follows that a sufficient condition for $\mathrm{m}_{\mathrm{o}}$ to be a point of local convexity is

$$
\xi_{0}=\nabla \varphi\left(m_{0}\right)+\tau m_{0}, \tau>-\kappa_{1} .
$$

In addition, it is immediate from (i) and (8.1) that if $\lambda \geq 0$ and $\varphi\left(\mathrm{m}_{0}\right) \leq \varphi(\mathrm{m})$ for all $|\mathrm{m}|=1$, then $\mathrm{m}_{\mathrm{o}}$ is a global point of convexity. Using (8.6) and (8.2), we conclude that $\mathrm{m}(\mathrm{x})=\mathrm{m}_{\mathrm{o}}$ is metastable if

$$
\mathrm{h}_{\mathrm{o}}=\mathrm{Dm}_{\mathrm{o}}+\nabla \varphi\left(\mathrm{m}_{\mathrm{o}}\right)+\tau \mathrm{m}_{\mathrm{o}}, \tau \in\left(-\kappa_{1}, \infty\right) .
$$

Brown assumes that $\nabla \varphi\left(\mathrm{m}_{\mathrm{o}}\right)=0$ and concludes that the single domain state $\mathrm{m}(\mathrm{x})=\mathrm{m}_{\mathrm{o}}, \mathrm{x} \in \Omega$, is metastable at least until $h_{0}$ reaches $D_{m_{0}}-\kappa_{1} m_{0}$. Since $\kappa_{1}$ is measured for the common ferromagnetic materials, this calculation provides a quantitative bound for the fields necessary to prevent a breakdown of the single domain state. As discussed by Brown [1963, p. 69-70], this 


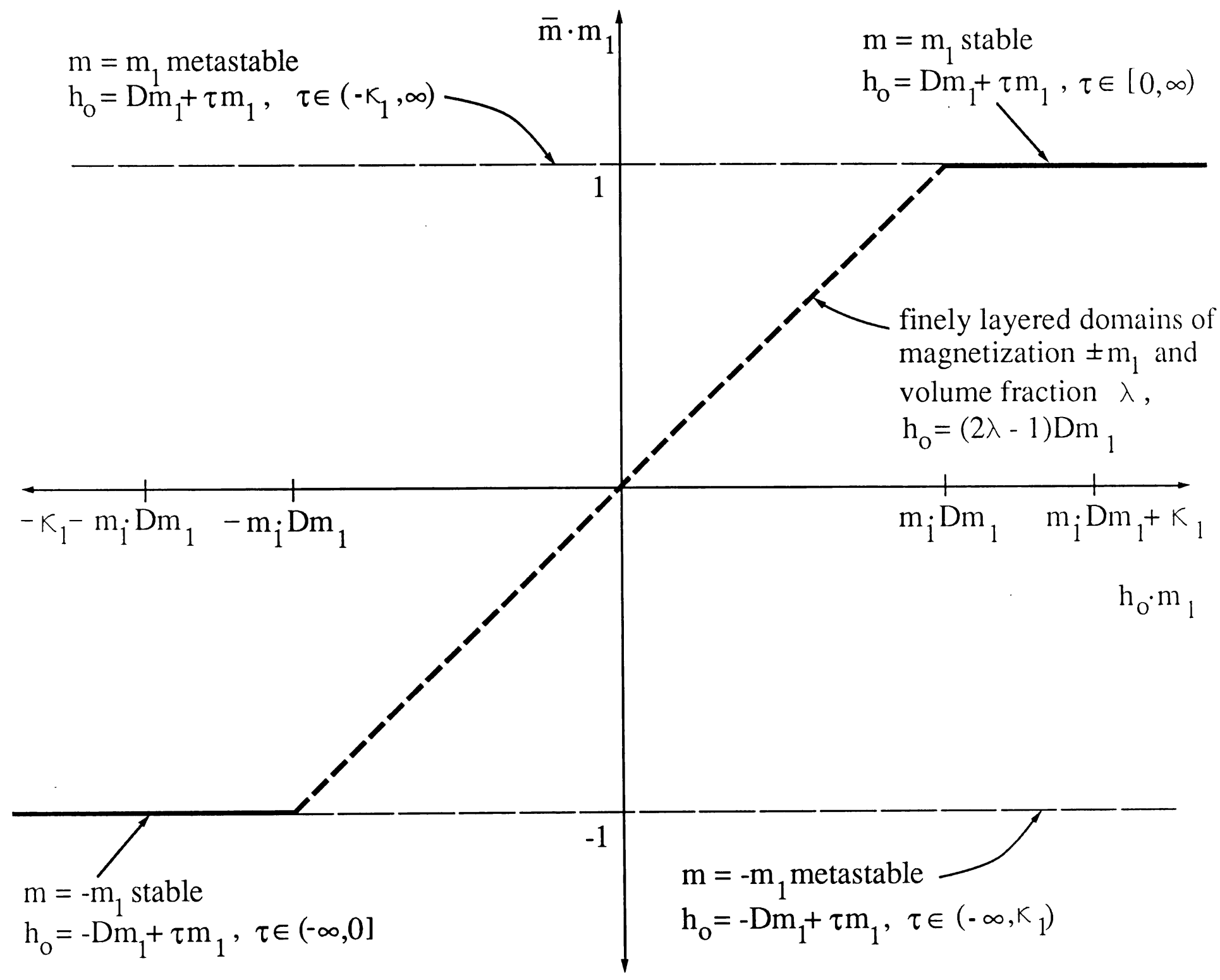

Figure 3. Curves showing average magnetization $v s$. field calculated from minimizers or relative minimizers of the total energy. Dark lines (-) correspond to absolute minimizers, dashed dark lines $(--)$ correspond to minimizing sequences, and dashed light lines $(--)$ correspond to relative minimizers in the sense of Brown. Fields $h_{o}$ corresponding to the various minima and relative minima are as indicated. In all cases $\Omega$ is an ellipsoid with demagnetizing matrix $D$ and $\pm \mathrm{m}_{1}$ minimize the anisotropy energy . 
bound is several orders of magnitude larger than that actually measured to produce breakdown. For example, in iron the calculation implies metastability for fields up to $\left|h_{0}\right| \doteq 500$ oe, while the measured value is clearly no greater than about $0.1 \mathrm{oe}$. This discrepancy is termed the coercivity paradox by Brown. Brown's results and our results of this section and Section 7 are summarized by Figure 3 .

A full discussion of the coercivity paradox can be found in Brown [1963, Sect. 5.2]. He argues that the paradox cannot be resolved by accounting for magnetostriction or by domain theory arguments. The general conclusion is that the paradox may be resolved by the inclusion of imperfections of some type, possibly even deviations from perfect ellipsoidal shape. We believe that part of the discussion is obscured by the lack of a clear description of what is the absolute minimizer, or in this case, the minimizing sequence. For example, the remark just before equation (8.7) implies that the single domain state is absolutely stable only up to the field $h_{o}=\mathrm{Dm}_{1}$ and it is possible that a more careful consideration of both stable and metastable states may yield a resolution to the coercivity paradox. A full analysis of these remarks will be found in a forthcoming paper.

We now turn to a discussion of the paper of Lifshitz [1944] which proposes, from the point of view of domain theory, the splitting of layered domains near the boundary. At first, this proposal resembles our results of Section 5 on the necessary splitting of domains near the boundary in cubic materials, but the splitting proposed by Lifshitz occurs on (100) planes and therefore is unrelated to our calculation. In fact, the splitting predicted by Lifshitz has origins in the magnetostrictive contribution to the energy. Lifshitz' calculations are highly suggestive that in a setting that includes magnetostriction, e.g. $\varphi(\mathrm{m}, \nabla \mathrm{y})$, where $\mathrm{y}: \Omega \rightarrow \mathbb{R}^{3}$ represents the deformation, the minimum energy state would not be attained. The case ${ }^{*} \varphi(m, \nabla y)$ has also been

\footnotetext{
* As written, we have in mind that the exchange energy is omitted, the case treated by most authors. Interestingly, Lifshitz retains the exchange energy.
} 
treated extensively in the literature. If this suggestion is true, the various "equilibrium equations" found by putting the first variation of the total magnetostrictive energy equal to zero would not be useful for finding the minimum energy state.

Acknowledgements. We would like to thank Stuart Antman and Howard Savage for bringing to our attention the highly magnetostrictive $\mathrm{Tb}(\mathrm{Dy}) \mathrm{Fe}_{2}$ alloys which undergo domain refinement. Our attempt to understand this phenomenon motivated this study. We would also like to thank Mitchell Luskin for comments on the manuscript. We are pleased to acknowledge the support of the National Science Foundation and the Air Force Office of Scientific Research through NSF/DMS-8718881.

\section{References}

Anzellotti, G., S. Baldo and A. Visintin [1990] Asymptotic behavior of the Landau-Lifshitz model of ferromagnetism, Applied Mathematics and Optimization, to appear.

Ball, J.M. [1989] A version of the fundamental theorem for Young measures, Lecture Notes in Physics 344 (ed. M. Rascle, D. Serre and M. Slemrod), Springer-Verlag, 207-215.

Ball, J.M. and R.D. James [1987] Fine phase mixtures as minimizers of energy, Arch. Rat. Mech. Anal. 100, 1352.

Ball, J.M. and R.D. James [1990] Proposed experimental tests of a theory of fine microstructure and the two-well problem, preprint.

Brown, W.F. [1962] Magnetostatic Principles in Ferromagnetism, Vol. 1 of Selected Topics in Solid State Physics (ed. E.P. Wohlfarth), North-Holland.

Brown, W.F. [1963] Micromagnetics, John Wiley and Sons, New York.

Brown, W.F. [1966] Magnetoelastic Interactions, Vol. 9 of Springer Tracts in Natural Philosophy (ed. C. Truesdell), Springer-Verlag.

Carey, R. and E.D. Isaac [1966] Magnetic Domains and Techniques for their Observation, Academic Press.

Chikazumi, S. [1964] Physics of Magnetism (trans. S.H. Charap), John Wiley and Sons, New York. 
Chipot, M. and D. Kinderlehrer [1988] Equilibrium configurations of crystals, Arch. Rat. Mech. Anal. 103, 237277.

Collins, C. and M. Luskin [1989] The computation of the austenitic-martensitic phase transition, Lecture Notes in Physics 344 (ed. M. Rascle, D. Serre and M. Slemrod), Springer-Verlag, 34-50.

Craik, D.J. and R.S. Tebble [1965] Ferromagnetism and Ferromagnetic Domains, North-Holland.

Dacorogna, B. [1982] Weak continuity and weak lower semicontinuity of nonlinear functionals, Springer Lecture Notes in Math. 922, Springer-Verlag, New York.

Ekeland, I. and R. Temam [1976] Convex Analysis and Variational Problems, North-Holland.

Fonseca, I. [1988] The lower quasiconvex envelope of the stored energy function for an elastic crystal, J. Math. pures et appl. 67, 175-195.

Frank, F.C. [1958] On the theory of liquid crystals, Discussions Faraday Soc. 25, 19-28.

James, R.D. [1989] Relation between microscopic and macroscopic properties of crystals undergoing phase transformation, in Proc. 7th Army Conf. on Applied Mathematics and Computing (ed. F. Dressel).

James, R.D. and D. Kinderlehrer [1989] Theory of diffusionless phase transformations, Lecture Notes in Physics 344 (ed. M. Rascle, D. Serre and M. Slemrod), Springer-Verlag, 51-84.

Kléman, M. [1983] Points, Lines and Walls, John Wiley and Sons, New York.

Kohn, R.V. [1989] The relationship between linear and nonlinear variational models of coherent phase transitions, in Proc. 7th Army Conf. on Applied Mathematics and Computing (ed. F. Dressel).

Kohn, R.V. and P. Sternberg [1989] Local minimisers and singular perturbations, Proc. Royal Soc. Edinburgh 111A, 69-84.

Landau, L.D. and E.M. Lifshitz [1935] Physik. Z. Sowjetunion 8, 337-346.

Landau, L.D. and E.M. Lifshitz and L.P. Pitaevskii. [1984] Electrodynamics of Continuous Media, Vol. 8 of Course of Theoretical Physics, Pergamon Press.

Lifshitz, E.M. [1944] On the magnetic structure of iron, J. Physics 8, 337-346.

Moreau, J.-J. [1964] Théorèms 'inf-sup', C. R. Acad. Sci. Paris 258, 2720-2722.

Osborn, F.A. [1945] Demagnetizing factors of the general ellipsoid, Phys. Rev. 67, 351-357.

Pedregal [1989] Thesis, University of Minnesota.

Rogers, R.C. [1988] Nonlocal variational problems in nonlinear electromagneto-elastostatics, SIAM J. Math. Anal. 19, 1329-1347.

Sethna, J. [1987] Theory of the blue phases of chiral nematic liquid crystals, in Theory and Applications of Liquid Crystals (ed. J.L. Ericksen and D. Kinderlehrer), Vol. 5 of The IMA Volumes in Mathematics and Its Applications, Springer-Verlag, New York, 305-324

Stampacchia, G. [1964] Formes bilinéaires coercitives sur les ensembles convexes, C. R. Acad. Sci. Paris 258, $4413-4416$. 
Stoner, E.C. [1945] The demagnetizing factors for ellipsoids, Phil. Mag. 36, 803-821.

Tartar, L. [1984] Ètude des oscillations dans les équations aux dérivées partielles nonlinéares, Springer Lecture
Notes in Physics 195, 384-412. Young, L.C. [1980] Lectures on Calculus of Variations and Optimal Control Theory, Chelsea. 
Li Ta-Tsien (Li Da-qian) and Zhao Yan-Chun, Global Existence of Classical Solutions to the Typical Free Boundary Problem for General Quasilinear Hyperbolic Systems and its Applications Thierry Cazenave and Fred B. Weissler, The Structure of Solutions to the Pseudo-Conformally Invariant Nonlinear Schrödinger Equation

Marshall Slemrod and Athanasios E. Tzavaras, A Limiting Viscosity Approach for the Riemann Problem in Isentropic Gas Dynamics

Richard D. James and Scott J. Spector, The Formation of Filamentary Voids in Solids

P.J. Vassiliou, On the Geometry of Semi-Linear Hyperbolic Partial Differential Equations in the Plane Integrable by the Method of Darboux

Jerome V. Moloney and Alan C. Newell, Nonlinear Optics

Keti Tenenblat, A Note on Solutions for the Intrinsic Generalized Wave and Sine-Gordon Equations

P. Szmolyan, Heteroclinic Orbits in Singularly Perturbed Differential Equations

Wenxiong Liu, A Parabolic System Arising In Film Development

Daniel B. Dix, Temporal Asymptotic Behavior of Solutions of the Benjamin-Ono-Burgers Equation

Michael Renardy and Yuriko Renardy, On the nature of boundary conditions for flows with moving free surfaces

Werner A. Stahel, Robust Statistics: From an Intellectual Game to a Consumer Product

Avner Friedman and Fernando Reitich, The Stefan Problem with Small Surface Tension

E.G. Kalnins and W. Miller, Jr., Separation of Variables Methods for Systems of Differential Equations in Mathematical Physics

Mitchell Luskin and George R. Sell, The Construction of Inertial Manifolds for Reaction-Diffusion Equations by Elliptic Regularization

Konstantin Mischaikow, Dynamic Phase Transitions: A Connection Matrix Approach

Philippe Le Floch and Li Tatsien, A Global Asymptotic Expansion for the Solution to the Generalized Riemann Problem

Matthew Witten, Ph.D., Computational Biology: An Overview

Matthew Witten, Ph.D., Peering Inside Living Systems: Physiology in a Supercomputer

Michael Renardy, An existence theorem for model equations resulting from kinetic theories of polymer solutions

Daniel D. Joseph and Luigi Preziosi, Reviews of Modern Physics: Addendum to the Paper "Heat Waves"

Luigi Preziosi, An Invariance Property for the Propagation of Heat and Shear Waves

Gregory M. Constantine and John Bryant, Sequencing of Experiments for Linear and Quadratic Time Effects

Prabir Daripa, On the Computation of the Beltrami Equation in the Complex Plane

Philippe Le Floch, Shock Waves for Nonlinear Hyperbolic Systems in Nonconservative Form

A.L. Gorin, D.B. Roe and A.G. Greenberg, On the Complexity of Pattern Recognition Algorithms On a Tree-Structured Parallel Computer

Mark J. Friedman and Eusebius J. Doedel, Numerical computation and continuation of invariant manifolds connecting fixed points

Scott J. Spector, Linear Deformations as Global Minimizers in Nonlinear Elasticity

Denis Serre, Richness and the classification of quasilinear hyperbolic systems

L. Preziosi and F. Rosso, On the stability of the shearing flow between pipes

Avner Friedman and Wenxiong Liu, A system of partial differential equations arising in electrophotography

Jonathan Bell, Avner Friedman, and Andrew A. Lacey, On solutions to a quasilinear diffusion problem from the study of soft tissue

David G. Schaeffer and Michael Shearer, Loss of hyperbolicity in yield vertex plasticity models under nonproportional loading

Herbert C. Kranzer and Barbara Lee Keyfitz, A strictly hyperbolic system of conservation laws admitting singular shocks

S. Laederich and M. Levi, Qualitative dynamics of planar chains

Milan Miklavčič , A sharp condition for existence of an inertial manifold

Charles Collins, David Kinderlehrer, and Mitchell Luskin, Numerical approximation of the solution of a variational problem with a double well potential

Todd Arbogast, Two-phase incompressible flow in a porous medium with various nonhomogeneous boundary conditions 
Title

Peter Poláčik, Complicated dynamics in scalar semilinear parabolic equations in higher space dimension

Bei Hu, Diffusion of penetrant in a polymer: a free boundary problem

Mohamed Sami ElBialy, On the smoothness of the linearization of vector fields near resonant hyperbolic rest points

Max Jodeit, Jr. and Peter J. Olver, On the equation $\operatorname{grad} f=M \operatorname{grad} g$

Shui-Nee Chow, Kening Lu, and Yun-Qiu Shen, Normal form and linearization for quasiperiodic systems

Prabir Daripa, Theory of one dimensional adaptive grid generation

Michael C. Mackey and John G. Milton, Feedback, delays and the origin of blood cell dynamics

D.G. Aronson and S. Kamin, Disappearance of phase in the Stefan problem: one space dimension

Martin Krupa, Bifurcations of relative equilibria

D.D. Joseph, P. Singh, and K. Chen, Couette flows, rollers, emulsions, tall Taylor cells, phase separation and inversion, and a chaotic bubble in Taylor-Couette flow of two immiscible liquids

Artemio González-López, Niky Kamran, and Peter J. Olver, Lie algebras of differential operators in two complex variables

L.E. Fraenkel, On a linear, partly hyperbolic model of viscoelastic flow past a plate

Stephen Schecter and Michael Shearer, Undercompressive shocks for nonstrictly hyperbolic conservation laws

Xinfu Chen, Axially symmetric jets of compressible fluid

J. David Logan, Wave propagation in a qualitative model of combustion under equilibrium conditions

M.L. Zeeman, Hopf bifurcations in competitive three-dimensional Lotka-Volterra Systems

Allan P. Fordy, Isospectral flows: their Hamiltonian structures, Miura maps and master symmetries

Daniel D. Joseph, John Nelson, Michael Renardy, and Yuriko Renardy, Two-Dimensional cusped interfaces

Avner Friedman and Bei $\mathbf{H u}$, A free boundary problem arising in electrophotography

Hamid Bellout, Avner Friedman and Victor Isakov, Stability for an inverse problem in potential theory

Barbara Lee Keyfitz, Shocks near the sonic line: A comparison between steady and unsteady models for change of type

Barbara Lee Keyfitz and Gerald G. Warnecke, The existence of viscous profiles and admissibility for transonic shocks

P. Szmolyan, Transversal heteroclinic and homoclinic orbits in singular perturbation problems

Philip Boyland, Rotation sets and monotone periodic orbits for annulus homeomorphisms

Kenneth R. Meyer, Apollonius coordinates, the N-body problem and continuation of periodic solutions

Chjan C. Lim, On the Poincare-Whitney circuitspace and other properties of an incidence matrix for binary trees

K.L. Cooke and I. Györi, Numerical approximation of the solutions of delay differential equations on an infinite interval using piecewise constant arguments

Stanley Minkowitz and Matthew Witten, Periodicity in cell proliferation using an asynchronous cell population

M. Chipot and G. Dal Maso, Relaxed shape optimization: The case of nonnegative data for the Dirichlet problem

Jeffery M. Franke and Harlan W. Stech, Extensions of an algorithm for the analysis of nongeneric Hopf bifurcations, with applications to delay-difference equations

Xinfu Chen, Generation and propagation of the interface for reaction-diffusion equations

Philip Korman, Dynamics of the Lotka-Volterra systems with diffusion

Harlan W. Stech, Generic Hopf bifurcation in a class of integro-differential equations

Stephane Laederich, Periodic solutions of non linear differential difference equations

Peter J. Olver, Canonical Forms and Integrability of BiHamiltonian Systems

S.A. van Gils, M.P. Krupa and W.F. Langford, Hopf bifurcation with nonsemisimple 1:1 Resonance

R.D. James and D. Kinderlehrer, Frustration in ferromagnetic materials

Carlos Rocha, Properties of the attractor of a scalar parabolic P.D.E.

Debra Lewis, Lagrangian block diagonalization 